\title{
Aplicaciones de la termocronología por trazas de fisión en Venezuela: Estado actual, perspectivas y visión integradora
}

\author{
Mauricio Alberto Bermúdez ${ }^{1 *}$, Marlene Flores-Ferrín ${ }^{2}$ \\ doi: http://dx.doi.org/10.18273/revbol.v41n3-2019009 (c) (1) \\ Forma de citar: Bermúdez, M.A., y Flores-Ferrín, M. (2019). Aplicaciones de la termocronología por trazas de \\ fisión en Venezuela: Estado actual, perspectivas y visión integradora. Boletín de Geología, 41(3), 175-200. doi: \\ 10.18273/revbol.v41n3-2019009
}

\section{RESUMEN}

La Termocronología por Trazas de Fisión (TTF) se basa en el decaimiento radiactivo por fisión nuclear espontánea de los átomos de ${ }^{238} \mathrm{U}$. Estos átomos están presentes en minerales accesorios tales como apatito, circón, y esfena, especies minerales en varios tipos de rocas. Este tipo de fisión produce unas zonas de daño en la estructura cristalina de los minerales conocida como trazas de fisión, estructuras lineales cortas de ca. 14-18 $\mu \mathrm{m}$ y entre 1 a 4 micrones de diámetro que son preservadas al pasar ciertas isotermas (dependiendo de las diferentes especies minerales), pero generalmente en rangos de temperatura inferiores a $\operatorname{los} 300^{\circ} \mathrm{C}$. El rango de aplicaciones de la TTF es muy amplio e incluye investigaciones en los campos de: a) arqueología (datación de vasijas, fósiles, paleo-incendios); b) la evolución tectono-térmica de áreas geológicamente complejas; c) la discriminación de interacciones entre clima, tectónica, y procesos superficiales; y d) la evolución termal de las principales cuencas petrolíferas. Desde la fundación del Laboratorio de Termocronología de la Universidad Central de Venezuela por parte de Petróleos de Venezuela, SA (PDVSA) en 1995 ha apoyado muchas investigaciones en todo el país que aplican esta técnica y especialmente en la región de los Andes venezolanos. En este trabajo, se realiza una revisión de los distintos estudios por TTF que se han desarrollado hasta ahora en Venezuela, y se proporcionan las posibles aplicaciones a futuro y proponemos algunos problemas que aún quedarían pendientes por resolver.

Palabras clave: Termocronología; trazas de fisión; aplicaciones en Venezuela; tectónica; clima; exhumación.

\section{Applications of fission-track thermochronology in Venezuela: Current status, perspectives and integrative approaches}

\begin{abstract}
Fission-Track Thermochronology (FTT) is based on the radioactive decay by spontaneous nuclear fission of ${ }^{238} \mathrm{U}$ atoms. These are present in accessory minerals such as apatite, zircon, and titatine contained in various types of rocks. The spontaneous fission produces damage, known as fission-track, to the crystalline structure of minerals. These tracks are short linear structures of ca. 14-18 $\mu \mathrm{m}$ and between 1-4 microns of diameter and become preserved when crossing certain isotherms (depending on the mineral species), but generally in ranges below $300^{\circ} \mathrm{C}$. The range of applications of FTT is very wide and includes research in the field of: a) archaeology (dating of vessels, fossils, paleo-fire); b) tectonic-thermal evolution of geologically complex areas; c) climate, tectonics, and surface processes, and their interactions; and d) the thermal evolution of main oil basins. Since its foundation in 1995, the Laboratory of Thermochronology in the Central University of Venezuela (established by Petróleos de Venezuela SA - PDVSA) has supported much research throughout the country applying this technique and especially in the region of the Venezuelan Andes. In this paper, we present a review of research developed in Venezuela based on FTT, discussing fields of high potential for future applications, and challenging pending to solve.
\end{abstract}

Keywords: Thermochronology; fission-track; applications in Venezuela; tectonics; climate; exhumation.

${ }^{1}$ Escuela de Ingeniería Geológica, Universidad Pedagógica y Tecnológica de Colombia, Sogamoso, Colombia. (*)mauricio.bermudez@uptc.edu.co

${ }^{2}$ Laboratorios de Termocronología y Geomatemática, Escuela de Geología, Minas y Geofísica, Universidad Central de Venezuela, Caracas, Venezuela. marlenefferrin@gmail.com 


\section{INTRODUCCIÓN}

La termocronología de baja temperatura (trazas de fisión y $(\mathrm{U}-\mathrm{Th}) / \mathrm{He}$ ) se basa en el decaimiento radioactivo de isotopos tales como uranio-238 $\left({ }^{238} \mathrm{U}\right)$, torio-232 $\left({ }^{232} \mathrm{Th}\right)$, entre otros, permitiendo extraer la historia termal de las rocas (Braun et al., 2006). Cada técnica mide la concentración y relación de isótopos padres y productos hijos de una forma específica, utilizando espectrómetros de masas y otros equipos especializados. La termocronología se basa en la interacción entre la acumulación de un producto hijo, el cual puede ser o bien un isótopo, o bien un defecto en la estructura cristalina del mineral, formado por el decaimiento radioactivo dentro de un mineral en específico y la eliminación de ese isótopo hijo por la activación de un mecanismo de difusión termal (Wagner y van den Haute, 1992).

Existen diversos métodos geocronológicos con diferentes aplicaciones (FIGURA 1). Estos métodos pueden ser usados en una amplia variedad de contextos geológicos y con diferentes filosofías de muestreo, por ejemplo, el método uranio-plomo $(\mathrm{U} / \mathrm{Pb})$ es aplicado con frecuencia a dos minerales: circón y monacita, ambos arrojan información sobre los eventos de enfriamiento magmático o procesos metamórficos. En la parte intermedia de la FIGURA 1 se encuentran los métodos termocronológicos de alta, media y baja temperatura consistente en rangos entre $450-600^{\circ} \mathrm{C}$, $250-450^{\circ} \mathrm{C}$ y 60 a $250^{\circ} \mathrm{C}$, respectivamente. En la parte baja de la figura, desde los $250^{\circ} \mathrm{C}$ aproximadamente, se encuentran los termocronómetros de baja temperatura. Actualmente, gracias a la termocronología cuantitativa (Braun et al., 2006) estos datos pueden ser usados específicamente para: 1) cuantificación numérica y simulación de cinturones de montañas activos desde la cristalización de las rocas hasta los procesos superficiales; 2) análisis termal de cuencas petrolíferas; 3) relación entre exhumación, tectónica y procesos de superficie; 4) evolución geodinámica de cinturones de montaña activos (e.g., Andes, Himalayas, Alpes, entre otros), y 5) modelos de la evolución del paisaje.

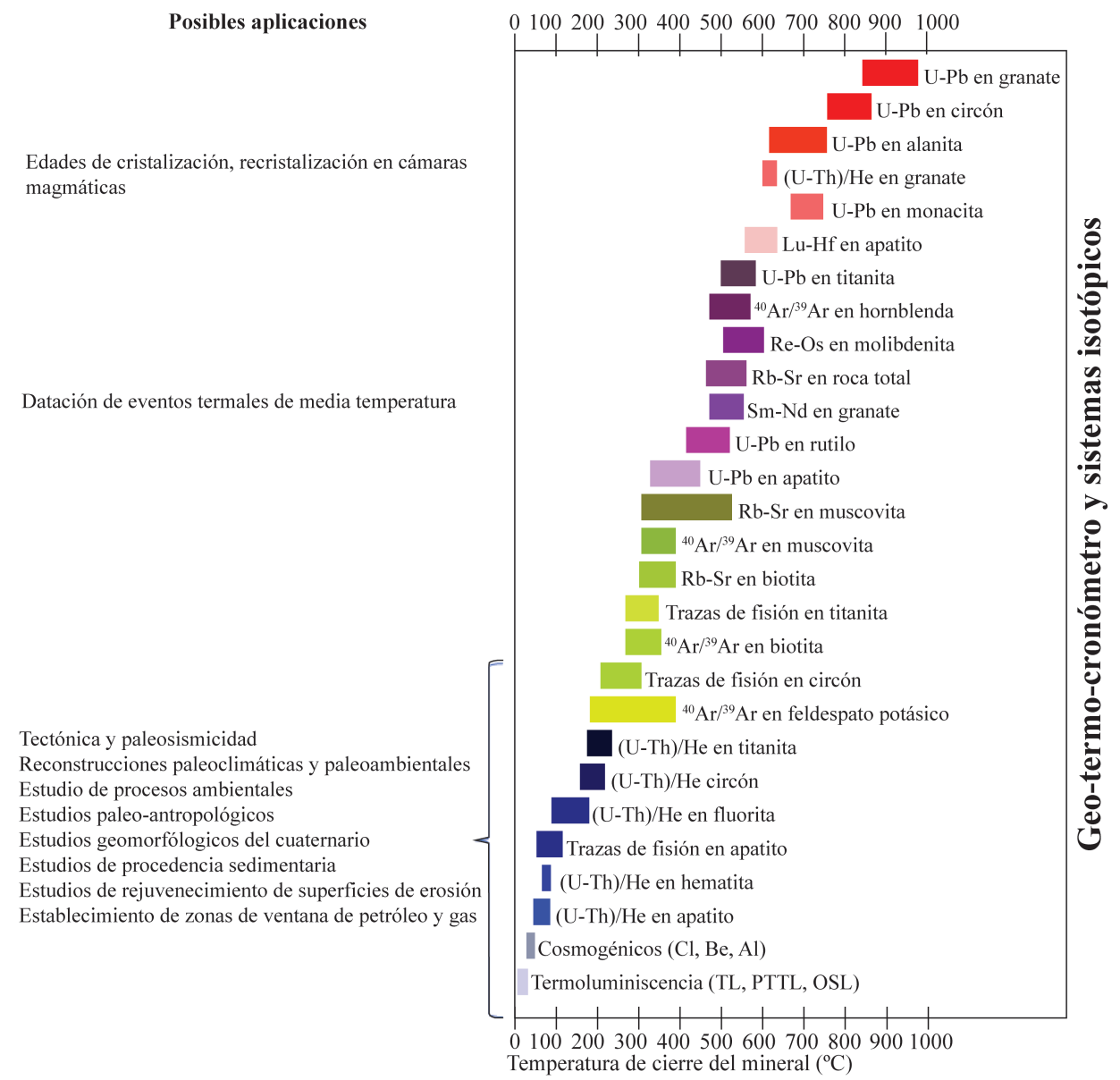

FIGURA 1. Posibles aplicaciones de los métodos geocronológicos y termocronológicos existentes (modificado a partir de Farley, 2002; Chew y Spikings, 2015). 
El concepto más importante para la aplicación de tales técnicas es el de temperatura de cierre (closure temperature; Dodson, 1973), definida como la temperatura mínima a la cual los minerales comienzan a retener los isótopos hijos producto del decaimiento radiactivo (Wagner y van den Haute, 1992). Algunos eventos geológicos pueden producir exhumación o soterramiento de rocas, disminuyendo o incrementando la temperatura de éstas, respectivamente. En esos movimientos, las rocas atraviesan ciertos rangos de isotermas, y a temperaturas inferiores a ellas, la información isotópica de productos radiogénicos se preserva.
Las isotermas son sensibles a la actividad hidrotermal, circulación de fluidos y actividad de las fallas. Ellas se comprimen debido a la advección del calor cerca de las zonas de fallas bien sea por la actividad de la falla o por la circulación de fluidos cerca de éstas, adicionalmente las isotermas son sensibles a los procesos de enfriamiento por erosión de las cadenas montañosas $\mathrm{y}$ al calentamiento por soterramiento de las cuencas sedimentarias. Un ejemplo de la advección de isotermas es representado en la FIGURA 2, en ella las líneas punteadas rojas representan algunas de las isotermas de cierre de cuatro métodos de termocronología de baja temperatura (AHe, AFT, ZHe y ZFT).

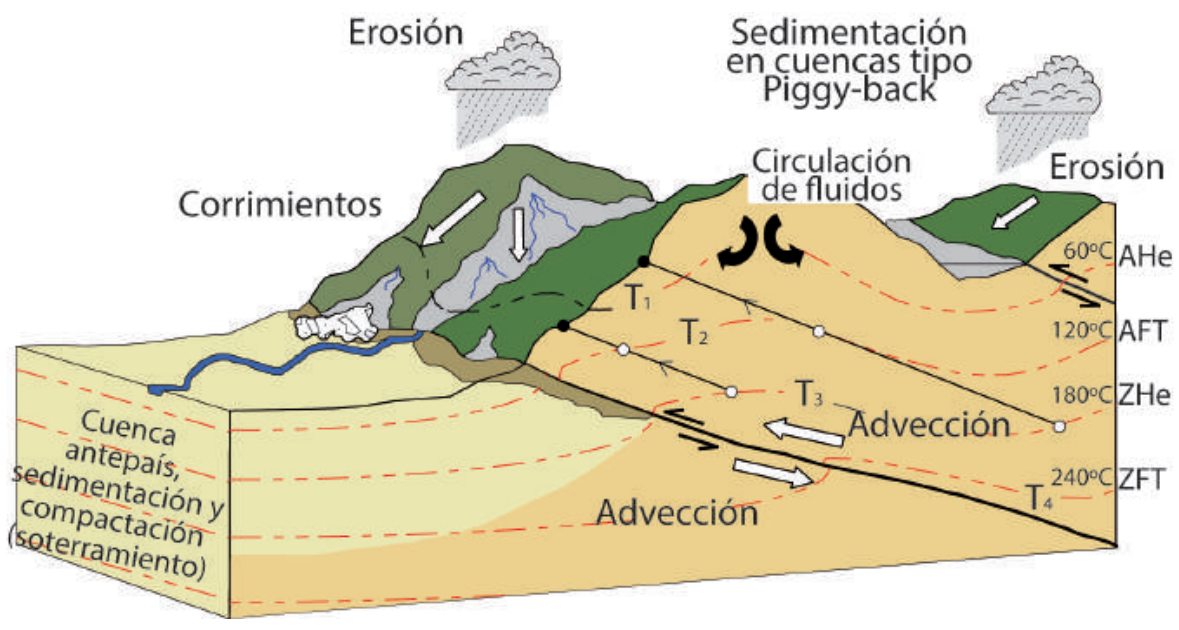

FIGURA 2. Isotermas de cierre $\left(\mathrm{T}_{1}\right.$ a $\left.\mathrm{T}_{4}\right)$ para distintos termocronómetros: AFT y ZFT corresponde al método de trazas de fisión en apatitos y circón, respectivamente, AHe y ZHe corresponde al método (U-Th)/He en apatito y circón, respectivamente y sensibilidad de estas a procesos de fallamiento, circulación de fluidos, erosión y soterramiento (tomada y modificada de Ehlers, 2005).

En este trabajo se presenta de forma muy resumida algunos aspectos teóricos fundamentales de la Termocronología por Trazas de Fisión (TTF), bajo la modalidad del Método de Detector Externo (EDM, por sus siglas en inglés) en apatitos y circones que ha sido una de las técnicas más utilizadas en Venezuela para conocer la historia de exhumación de algunas cadenas montañosas en el país, y la evolución tectonotermal de cuencas sedimentarias. Adicionalmente, se presenta una revisión de las principales aplicaciones de la técnica de trazas de fisión bajo la modalidad EDM en Venezuela, el estado actual y las perspectivas a futuro. A lo largo del presente documento denotaremos las edades por trazas de fisión en apatitos como AFT, en circón como ZFT y en esfena como SFT.

\section{FUNDAMENTOS TEÓRICOS DE LA TERMOCRONOLOGÍA POR TRAZAS DE FISIÓN}

El decaimiento radioactivo de los átomos de ${ }^{238} \mathrm{U}$ puede producirse por distintos mecanismos, entre los cuales destacan: emisión de partículas alfa $(\alpha)$, emisión de partículas beta ( $\beta$ ) y fisión nuclear (Faure, 1986). Aunque sólo una pequeña proporción de átomos de ${ }^{238} \mathrm{U}$ decae por fisión nuclear, este es el mecanismo más importante para la TTF. Durante la fisión nuclear, dos nuevos núcleos cargados positivamente con alta energía se desplazan en sentidos opuestos a gran velocidad. En la trayectoria de ambos fragmentos se produce una serie de repulsiones electrostáticas que 
generan una zona de daño en la estructura cristalina del mineral conocida como traza de fisión (Wagner y van den Haute, 1992). En la FIGURA 3, se presenta el modelo de explosión de iones o Ion explosion spike model (Fleischer et al., 1969) el cual trata de explicar la formación de una traza de fisión.

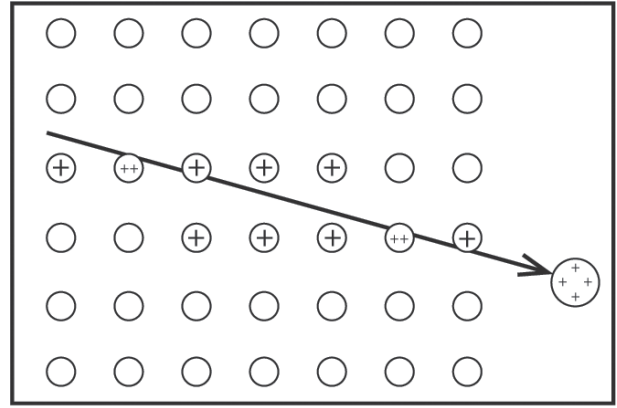

A.

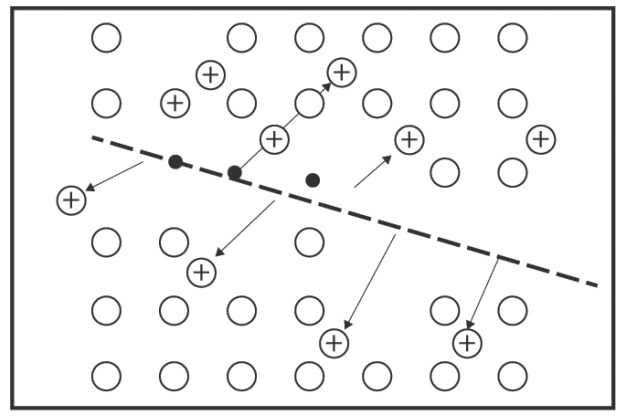

B.

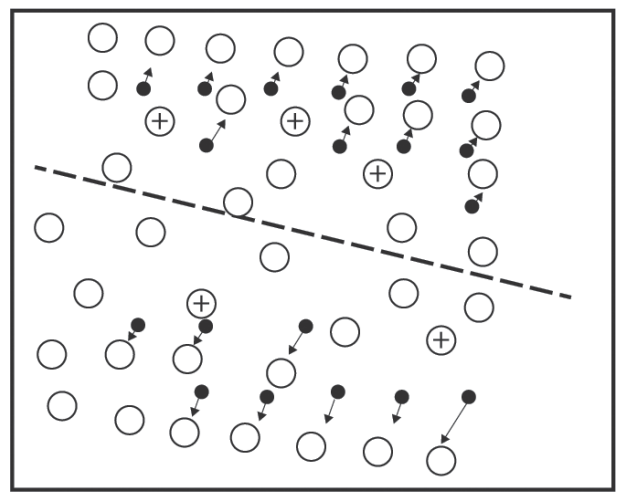

C.

FIGURA 3. Formación de una traza de fisión de acuerdo con la teoría ion explosion spike model. Para la formación al menos tres etapas son necesarias: A. Ionización de átomos de la red cristalina, por el movimiento altamente energético de las partículas cargadas. B. Los grupos de iones positivos resultantes se desplazan de sus sitios de red originales como resultado de la repulsión de Coulomb. C. La región afectada se relaja elásticamente, forzando la región circundante del sólido (tomada y modificada de Fleischer et al., 1969).

\section{Método del Detector Externo (EDM)}

El EDM consiste en la colocación de una mica o detector de bajo contenido de uranio al conjunto de minerales montados en una sección epóxica para el caso de los apatitos o sobre el teflón en el caso del circón. En la FIGURA 4 se observan las trazas de fisión en un cristal de circón y apatito después de haber sido concentrados los minerales y después del revelado de las trazas de fisión por ácido nítrico $\mathrm{HNO}_{3}$ para el caso del apatito y una solución eutéctica de hidróxido de potasio $(\mathrm{KOH})$ e hidróxido de sodio $(\mathrm{NaOH})$ para el circón. Al lado de cada uno de los minerales que se muestran en la FIGURA 4 se han colocado del lado derecho las respectivas micas (detector externo).

Los procesos necesarios para la datación de minerales por el método TTF bajo el EDM son mostrados en la FIGURA 5, en la parte A de esta, se muestra un contenedor hipotético donde se han dispuesto los vidrios dosímetros (de concentración de uranio conocido), las muestras estándares (de edad conocida por otros métodos de datación) y las muestras de edad desconocida $\left(M_{i}\right)$. En la FIGURA 5B se ha realizado un aumento de uno de los cristales que se encuentra en la sección epóxica de la FIGURA 5A. Previamente antes del montaje e irradiación, todos los cristales han pasado por un proceso previo de concentración mediante líquidos densos, pulido y revelado. Se dispone una mica sobre la superficie pulida y el paquete de muestras es irradiado con neutrones termales usando afluencias entre $10^{12}$ y $10^{15}$ neutrones $/ \mathrm{cm}^{2}$, esta variación depende del tipo de mineral y del grado de antigüedad que tengan. Se realiza la irradiación de estos con el fin de generar la fisión nuclear por inducción de los átomos de ${ }^{235} \mathrm{U}$ aún presentes en el interior del mineral. Cuando la muestra regresa al laboratorio, la mica es sumergida en HF usando una temperatura adecuada y durante 18 minutos con la finalidad de exponer las trazas de fisión inducidas. Posteriormente, se realiza un conteo de trazas de fisión espontánea e inducida en el montaje de minerales y en la mica, respectivamente, usando un microscopio óptico con aumento entre 1200 a 1600X.

Para determinar la edad desconocida $\left(t_{M}\right)$ de cada grano de apatito existente en el montaje de una muestra $M_{i}$ (ver FIGURA 5A) se emplea la siguiente ecuación:

$$
t_{M}=\frac{1}{\lambda_{\alpha}} \ln \left(\frac{\lambda_{\alpha}}{2} \frac{N_{s}}{N_{i}} \zeta \rho_{D}+1\right)
$$

donde $\lambda_{\alpha}=1,55125 \times 10^{-10}$ años $^{-1}$ es la constante de decaimiento de ${ }^{238} \mathrm{U}$ por emisión de partículas alfa, $N s, N i$ corresponde al número de trazas espontáneas e inducidas, respectivamente; $\zeta$ es conocido como el 


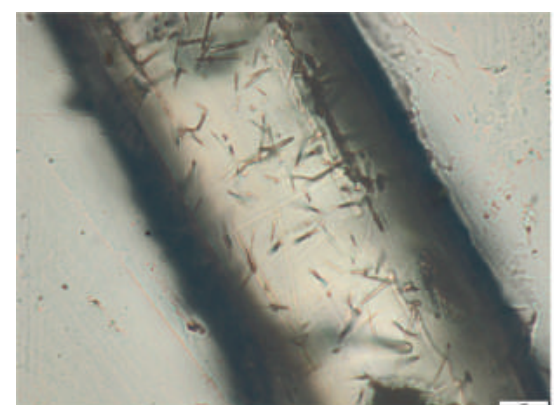

(A)

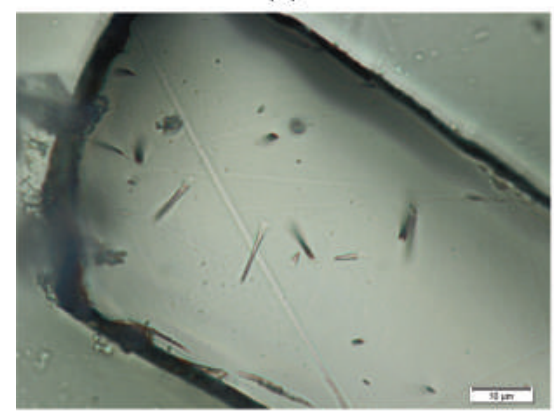

(B)

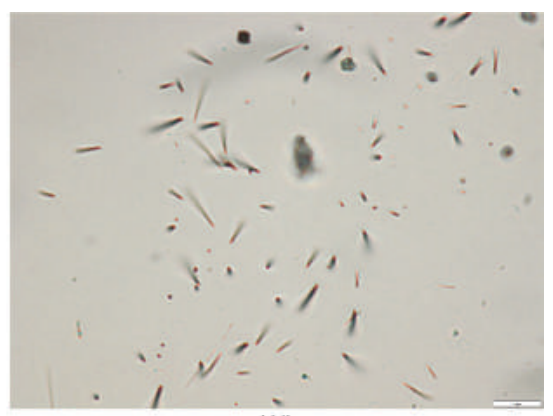

$\left(A^{\prime}\right)$

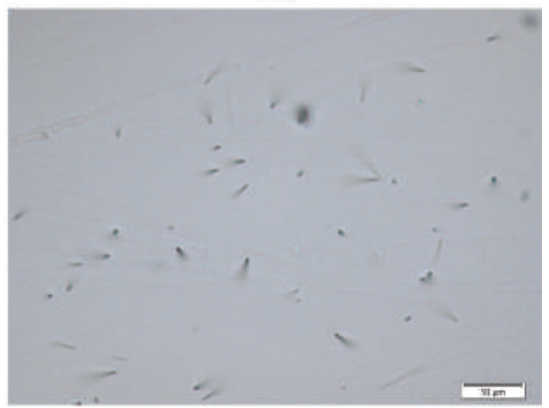

$\left(B^{\prime}\right)$

FIGURA 4. Trazas de fisión en diferentes minerales: A. Circón. A'. Mica sobre el circón. B. Apatito. B'. Mica sobre el apatito (imagen cortesía del Dr. Matthias Bernet, ISTERRE, Université Grenoble Alps, Francia).

(a)

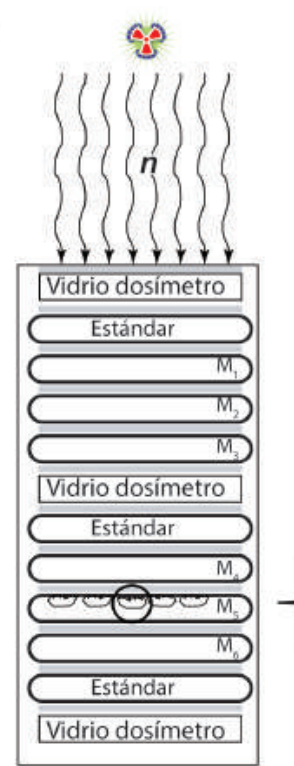

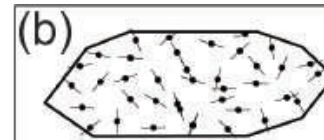
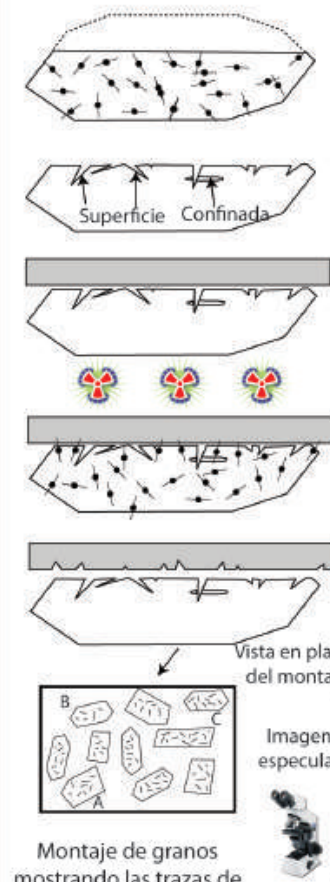

mostrando las trazas de

fisión espontáneas
Acumulación de trazas de fisión espontánea a lo largo del tiempo geológico

Sección pulida del cristal de apatito o circón

Revelado de trazas de fisión espontáneas

Colocación de la mica o detector externo

Irradiación termal con neutrones Registro de trazas de fisión inducidas en la mica

Revelado de trazas de fisión inducidas en la mica

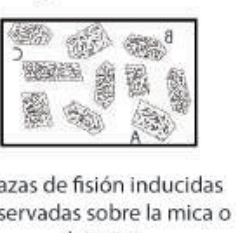
bservadas sobre la mica o detector

FIGURA 5. A. Representación esquemática de la posición de cada muestra de edad desconocida $M_{i}$, muestras estándares y dosímetros dentro de un contenedor al momento de ser irradiadas. B. Procesamiento de una muestra para su fechado mediante trazas de fisión modalidad EDM (modificado de Gallagher et al., 1998). 
factor de calibración y $\rho_{D}$ es la densidad de trazas en el vidrio o monitor de concentración de uranio conocida, es dado por el reactor nuclear al activar los átomos de uranio presentes en estos vidrios.
Seguidamente al cálculo de la edad, se determina el error (Galbraith, 2005) asociado a la edad utilizando la siguiente expresión:

$$
\sigma(t)=t \sqrt{\frac{1}{N_{S}+0,5}+\frac{1}{N_{i}+0,5}+\frac{1}{N_{D}}+\left(\frac{\sigma(\zeta)}{\zeta}\right)^{2}}
$$

En esta última expresión $N_{D}$ es la densidad de trazas calculadas sobre los vidrios dosímetros, y $\sigma(\zeta)$ es la desviación estándar del factor de calibración $\zeta$. Para efectos didácticos la derivación de la ecuación 1 y 2 pueden ser revisadas en Bermúdez et al. (2004, 2005) y en Wagner y van den Haute (1992).

$\mathrm{Al}$ momento de preparar las muestras en el contenedor para su irradiación, se intercalan varias secciones de apatitos o circones estándares o de edad conocida por otros métodos de datación como potasio-argón (K-Ar), rubidio-estroncio ( $\mathrm{Rb}-\mathrm{Sr}$ ), entre otros (ver FIGURA $5 \mathrm{~A}$ ). A estas secciones también se les realiza un conteo de trazas de fisión espontáneas e inducidas $N_{s}, N_{i}$, respectivamente, de la misma manera como se explicó anteriormente, con la diferencia de que, en lugar de calcular la edad, se estima el factor $\zeta$ presente en la ecuación 1. Entre los estándares más conocidos se utilizan: 1) Apatito de Durango con edades por K-Ar, de $31,4 \pm 0,5 \mathrm{Ma}$; 2) apatitos y circones de la toba volcánica Fish Canyon con edad por K-Ar de 27,8 40,5 $\mathrm{Ma}$; 3) circones del miembro Buluk con edades por $\mathrm{K}-\mathrm{Ar}$ de 16,4 $\pm 0,2 \mathrm{Ma}$; 4) circones de Dromedary

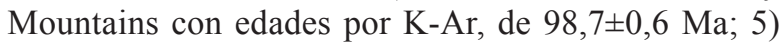
circones de la riolita de Tardree con edades por K-Ar, de $58,7 \pm 1,1 \mathrm{Ma}$; y 6) circones de la toba volcánica Bishop al este de California con edades por K-Ar, de 74,0 $\pm 0,6 \mathrm{Ma}$ (Hurford, 2019).

\section{APLICACIONES DE LA TERMOCRONOLOGÍA POR TRAZAS DE FISIÓN}

Existen diversas aplicaciones del método de TTF (Malusà y Fitzgerald, 2019). Sin embargo, en el presente artículo sólo se expone brevemente los fundamentos teóricos de dos de esas aplicaciones: 1) reconstrucción de la historia de enfriamiento (o historia térmica) de un determinado ambiente geológico y 2) reconstrucción termal de cuencas sedimentarias usando termocronología, ya que básicamente han sido las aplicaciones de la técnica en Venezuela. En las morrenas o depósitos glaciares en Venezuela no se han aplicado técnicas de termocronología por trazas de fisión.

\section{Historia de enfriamiento (o historia térmica) de un determinado ambiente geológico}

Los resultados obtenidos de densidad, longitud y distribución de longitud de trazas son aún parámetros físicos; por lo tanto, es necesario convertirlos en términos geológicos para determinar la edad y paleotemperatura de la muestra.

Las edades calculadas por el método TTF permiten datar la formación de rocas y minerales cuando se enfriaron a través de ciertas temperaturas de forma súbita, o pueden revelar la edad o intensidad de un evento térmico sobrepuesto o eventos sucesivos termales (Wagner y van den Haute, 1992); esto se refiere, a un evento geológico-térmico posterior que afectó térmicamente a las formaciones geológicas preexistentes.

Para encontrar la interpretación geológica apropiada, es necesario tomar en consideración el comportamiento (longitud) de las trazas de fisión según el tiempo y temperatura, ya que dentro de los diferentes parámetros geológicos (temperatura, presión, onda de choque, fluidos y radiación ionizante) ésta es una de las variables más importantes ya que puede ser relacionada con el tiempo, gracias a los conceptos de temperatura de cierre $\left(T_{c}\right)$ explicado anteriormente. El conjunto de límites de tiempo y temperatura en donde se alcanza la estabilidad parcial de la longitud de la traza es conocido como la Zona de Desvanecimiento Parcial (o en inglés Partial Annealing Zone: PAZ), esta última representa el comienzo de una descripción numérica del proceso de annealing que puede utilizarse para interpretar las edades TTF medidas en términos de la historia tectonotermal de una muestra (Hurford, 2019).

La reducción de la longitud de trazas por los procesos anteriormente mencionados es definida como annealing (Wagner y van den Haute, 1992). La distribución de frecuencias de longitudes de trazas (histogramas) arroja 
información importante acerca de la historia termal que la muestra ha experimentado. Algunos ejemplos de historia tiempo-Temperatura y la relación con la distribución de longitudes de trazas de fisión confinadas son mostradas en la FIGURA 6. Cuando una muestra hipotética ha sufrido un enfriamiento rápido desde $2 \mathrm{Ma}$ al presente (línea amarilla) el histograma de longitudes de trazas para este caso es unimodal, centrado hacia las longitudes largas (con una media de $16,3 \mu \mathrm{m}$, y desviación estándar de $0,9 \mu \mathrm{m})$. En el caso de la curva roja, la muestra reproduce un episodio volcánico rápido (expulsión piroclástica) ocurrida cerca de $20 \mathrm{Ma}$ se observa un rápido paso por la PAZ, hasta llegar a los $15^{\circ} \mathrm{C}$, posteriormente ya en superficie la roca que se enfría; el histograma resultante debería ser unimodal con longitud de traza promedio entre 14,5 a $15,0 \mu \mathrm{m}$ y una desviación estándar de 1,0 $\mu \mathrm{m}$. Esta distribución también se puede encontrar en rocas de origen no volcánico (basamento no perturbado, línea negra) y con una historia de enfriamiento relativamente rápido, seguido por la residencia a temperaturas más bajas (Gleadow et al., 1986; Green et al., 1986). La longitud promedio de las trazas de fisión estaría entre 14,5 y $15 \mu \mathrm{m}$ con una desviación estándar entre 1,2 y 2,0 $\mu \mathrm{m}$. Por último, la línea azul representa la historia termal de una muestra que ha sufrido enfriamiento a $20 \mathrm{Ma}$ hasta llegar a la superficie a $15 \mathrm{Ma}$, posteriormente un enfriamiento lento desde 15 a 9 Ma y calentamiento desde los 9 a $7 \mathrm{Ma}$, con una residencia dentro de la PAZ entre los 7 a $4 \mathrm{Ma}$, posteriormente un enfriamiento rápido desde los $4 \mathrm{Ma}$ al presente, los histogramas de distribuciones de longitudes de trazas tienden a ser bimodales o multimodales con longitudes de trazas promedios mayores a $11,5 \mu \mathrm{m}$ y desviaciones estándar mayores a 2,0 $\mu \mathrm{m}$ (Gleadow et al., 1986; Moore et al., 1986). Generalmente, cualquier roca que experimente en su historia tiempo-Temperatura episodios de enfriamiento-calentamiento va a tener un histograma parecido al bimodal o mixto (azul) y mientras más tiempo pase la muestra en la PAZ la longitud promedio de trazas de fisión tenderá a disminuir.

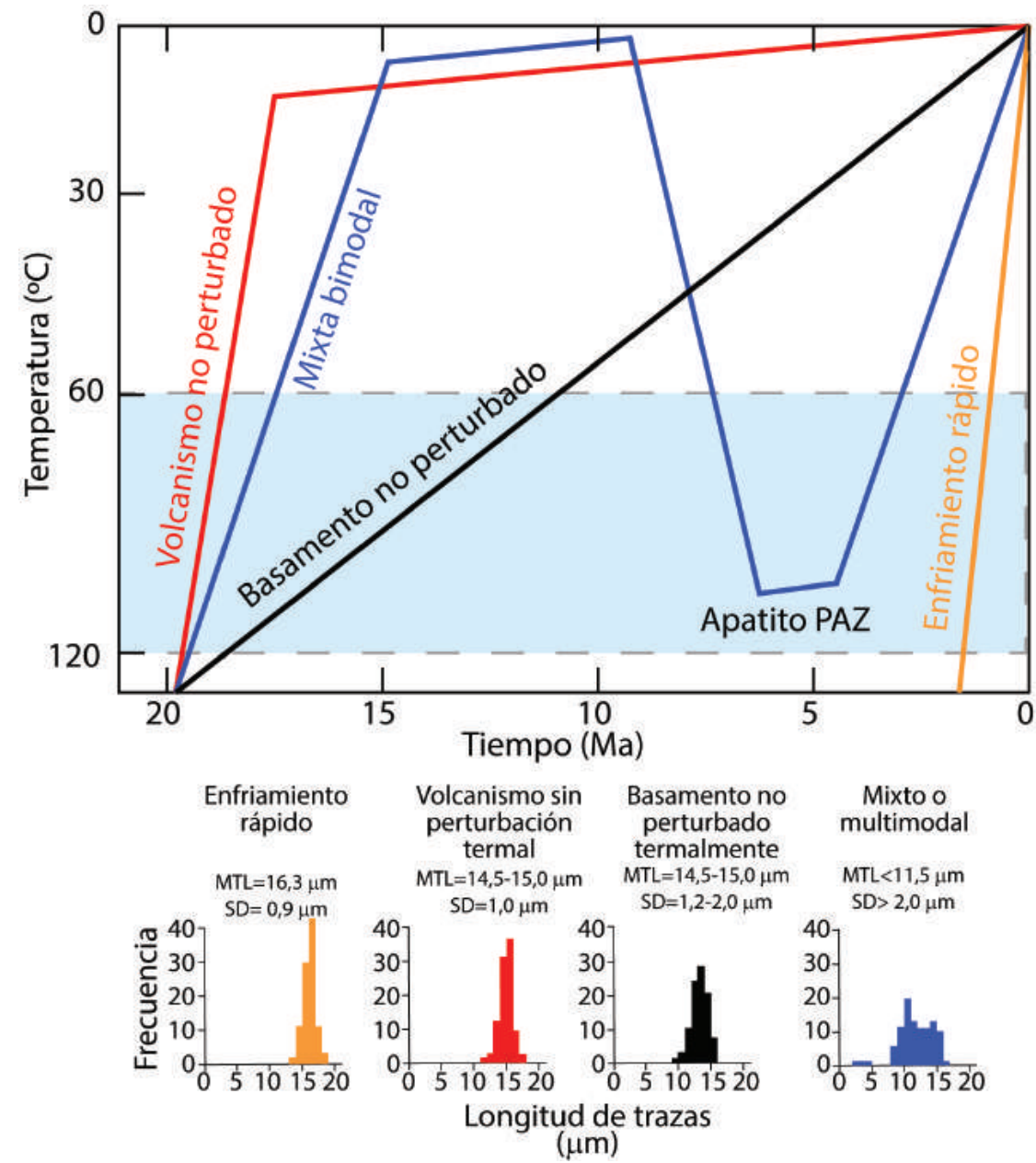

FIGURA 6. Influencia de la historia térmica sobre la acumulación de las trazas de fisión espontáneas (modificado de Hurford, 2019). MTL es la longitud promedio de trazas de fisión confinadas y SD se refiere a la desviación estándar. 
En resumen, si las muestras tienen una longitud promedio de traza de fisión corta y tienen grandes desviaciones estándar, entonces las muestras han pasado períodos prolongados de tiempo dentro de la PAZ, ya sea por enfriamiento monótono o por soterramiento posterior y consecuente exposición a altas temperaturas.

En la práctica es recomendable medir más de cien longitudes de trazas de fisión en apatitos sólo para una muestra. En caso de que la distribución de longitudes no sea suficiente, las muestras deben ser irradiadas en una fuente ${ }^{252} \mathrm{Cf}$ (Wagner y van den Haute, 1992) con la finalidad de incrementar la probabilidad de encontrar trazas confinadas.

\section{Reconstrucción termal de cuencas sedimentarias}

Entre los diversos factores geológicos que controlan la maduración del petróleo y gas en sedimentos se encuentra la temperatura. Para generar hidrocarburo líquido en condiciones naturales el sedimento debe ser soterrado hasta que entre en la llamada ventana de petróleo, entre $60 \mathrm{y} 130^{\circ} \mathrm{C}$ tal como puede apreciarse en la FIGURA 7, aparte de la máxima paleotemperatura, la evolución termal de los sedimentos también influye el proceso de maduración. Por casualidad, la temperatura de la ventana de petróleo coincide con el borrado de las trazas. Esto permite la reconstrucción de la historia termal de la cuenca sedimentaria por medio de análisis de trazas de fisión en apatitos. Dependiendo de su proveniencia geológica, los granos de apatitos individuales contienen varias cantidades de trazas de fisión espontáneas al momento de la sedimentación, una vez que la temperatura ha disminuido comienzan a generarse nuevas trazas de fisión, con el incremento de la subsidencia la temperatura se incrementa y a partir de aproximadamente $60^{\circ} \mathrm{C}$ comienzan a acortarse, el grado de acortamiento es muy sensible a la máxima temperatura alcanzada durante el calentamiento. Como el acortamiento afecta sólo a aquellas trazas que están presentes al momento del calentamiento, todas las trazas subsecuentes que se formen a bajas temperaturas deberán tener mayor longitud.

Un indicador independiente de madurez termal es la vitrinita, un componente orgánico del carbón (maceral) (Hood et al., 1975; Bostick et al., 1978; Dow, 1977, 1978; Teichmüller y Teichmüller, 1979). La reflectancia de la vitrinita $\left(\% \mathrm{R}_{0}\right)$ es una herramienta ampliamente utilizada para la evaluación de la historia termal en cuencas sedimentarias. La vitrinita es uno de los componentes primarios de los carbones y otras materias orgánicas que se encuentran en las rocas sedimentarias; se forma por la alteración térmica de la celulosa y la lignina en las paredes celulares de las plantas. A medida que este material se calienta, su capacidad de reflejar la luz aumenta. La reflectancia de la vitrinita registra así la temperatura máxima que una roca ha experimentado desde su enterramiento. No puede ser considerada como un termocronómetro, ya que no proporciona una edad ni ninguna otra indicación directa del tiempo. El estudio de la reflectancia de vitrinita (VR) es clave para identificar la historia de temperatura máxima alcanzada por los sedimentos en una cuenca. Los valores típicos de vitrinita están dados en unidades porcentuales de reflectancia $\left(\% \mathrm{R}_{0}\right)$ con valores que oscilan de $0 \%$ a $3 \%$ de $\mathrm{R}_{0}$. La TABLA 1 relaciona rangos de valores de reflectancia de vitrinita con la madurez termal y generación de hidrocarburos.

TABLA 1. Relación entre $\% \mathrm{R}_{0}$ y generación de hidrocarburos.

\begin{tabular}{cc}
\hline $\mathbf{\%}_{\mathbf{0}}$ & Madurez termal de la materia orgánica \\
\hline $\mathrm{R}_{0}<0,55$ & Inmaduro \\
$0,55<\mathrm{R}_{0}<0,80$ & Generación de petróleo y gas \\
$0,80<\mathrm{R}_{0}<1,0$ & Craqueo de petróleo a gas \\
$1,0<\mathrm{R}_{0}<2,5$ & Generación de gas seco \\
\hline
\end{tabular}

Valores de reflectancia de vitrinita cercanos al $0,7 \%$ corresponden a la temperatura bajo la cual todas las trazas de fisión en apatitos se desvanecen. Esto coincide con la precipitación de minerales de plomo y zinc en el Valle del Mississippi (MVT) por alteraciones hidrotermales (Wagner y van den Haute, 1992). Existe una relación entre la generación de hidrocarburos, la precipitación de minerales tipo MVT, la reflectancia de vitrinita, la cristalización de la illita/esmectita, el tipo de carbón e hidrocarburo y la reducción de la longitud de las trazas de fisión (ver FIGURA 7). 


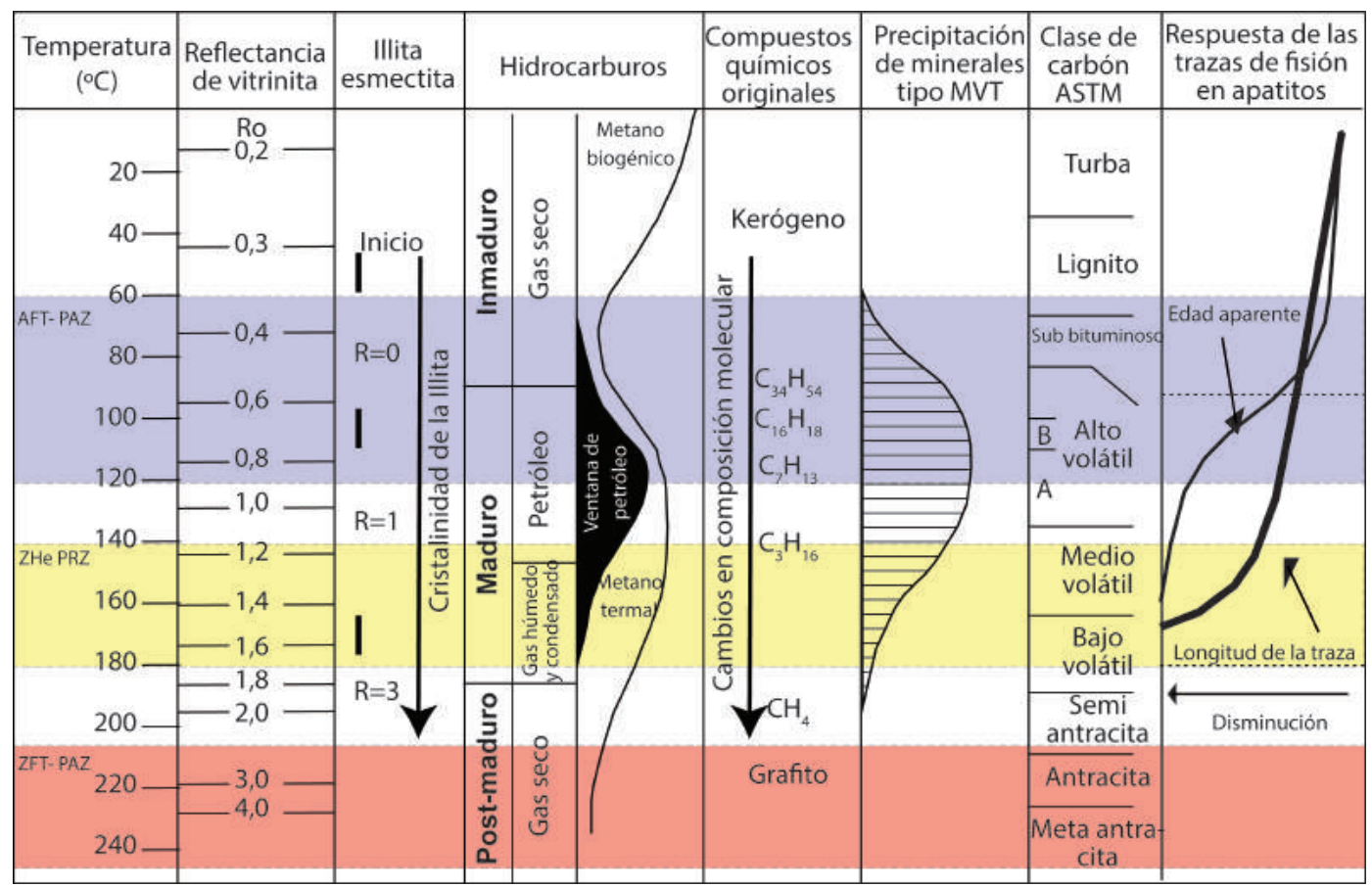

FIGURA 7. Comparación de temperaturas y profundidades para la generación de hidrocarburos, madurez y desvanecimiento de trazas de fisión en apatitos. Las franjas azules, amarillas y rojas se refieren a la PAZ para el método por trazas de fisión en apatitos, y la PRZ (Zona de Retención Parcial) para el método (U-Th)/He en circón y la zona de desvanecimiento parcial para el método por trazas de fisión en circón, respectivamente (modificada a partir de Wagner y van den Haute, 1992; Marshak, 2015).

Entre las principales aplicaciones del método de trazas de fisión en apatitos en la industria petrolera se tienen: 1) datación de la formación de trampas; 2) estimación del tiempo de exhumación de las cocinas para saber cuándo se dejó de generar petróleo (Mora et al., 2010, 2013); 3) estimación de la máxima temperatura alcanzada, e inferencia de la edad del proceso de generación.

La información anteriormente mencionada junto con datos de espesores, litología y edades permite establecer la historia termal de una cuenca, la cual tiene diversos beneficios, entre los cuales se destacan:

1.La reconstrucción de la historia termal de cuencas reduce significativamente el riesgo de exploración proporcionando información directa sobre el inicio de la generación de hidrocarburos.

2.Permite la definición confiable de áreas donde la generación de hidrocarburos toma lugar una vez existan y se conozca la evolución termocinemática de las trampas estructurales.
3.En áreas afectadas por múltiples episodios paleotermales, la información obtenida por el método de trazas de fisión aporta datos valiosos al estudio.

\section{APLICACIONES DE LA TERMOCRONOLOGÍA POR TRAZAS DE FISIÓN EN VENEZUELA}

La actual base de datos de edades obtenidas por el método de trazas de fisión en apatitos a lo largo de Venezuela puede ser observada en la FIGURA 8A. Las edades hasta ahora reportadas se concentran en la Sierra de Perijá, Andes de Venezuela, Cordillera de la Costa, Serranía del Interior, Montañas de Falcón y la península de Paria (FIGURA 8B). Estos sistemas montañosos son atravesados por importantes sistemas de fallas (FIGURA 8C) como lo son: Falla de Boconó y Falla de Valera en los Andes de Venezuela; fallas San Sebastián, La Victoria y Corrimiento Frontal en La Cordillera de la Costa, Falla de El Pilar y Falla de Urica en la Serranía del Interior y Falla El Tigre en la Sierra de Perijá. A continuación, se hace una breve revisión de las distintas publicaciones realizadas en el área. 


\section{Venezuela central y oriental: Cordillera de la Costa, Serranía del Interior y Península de Paria}

Las primeras dataciones se realizaron en la década de los ochenta por Kohn et al. (1984a), un total de 13 edades ZFT, 1 AFT y 1 por trazas de fisión en esfena fueron obtenidas. Las edades por trazas de fisión en circón oscilan entre $16,4 \pm 2,1$ y 49,0 $\pm 25,8 \mathrm{Ma}$. La edad AFT es de 6,1 11,3 Ma y la edad por trazas de fisión en esfena es de $126 \pm 15$ Ma. La principal conclusión de este estudio es que las edades por ZFT pueden ser interpretadas en un contexto amplio que permiten distinguir terrenos alóctonos de autóctonos. Las edades Eocenas son explicadas en términos de levantamiento, erosión y enfriamiento debido al rebote isostático. Once edades miocenas son relacionadas como un levantamiento regional que ocurre en respuesta de la translación lateral entre las placas suramericana y Caribe (FIGURA 8A). Los autores concluyen que es difícil dar una interpretación definitiva sobre el conjunto de datos.

Daly et al. (1995) reportaron 16 edades AFT en la Península de Paraguaná, montañas de Falcón (9 en la FIGURA 8A), estos autores sugieren que un enfriamiento comenzó entre 60 a $50 \mathrm{Ma}$, los cuales registran la colisión inicial de la Placa del Caribe con Suramerica. Combinando con reflectancia de vitrinita sugieren que la Península de Paraguaná no ha experimentado un enterramiento significativo y que ha permanecido como un bloque host elevado al presente. En contraste la región entre los Andes de Venezuela y la Cordillera de la Costa, llamada Andes de Barquisimeto al sur de Falcón si ha experimentado un enterramiento significativo, esa área pudo haber experimentado un evento reciente de enfriamiento entre 50 a $30 \mathrm{Ma}$.

Pérez de Armas (2005) reportó 48 edades por trazas de fisión en apatitos en los terrenos circundantes a la subcuenca de Guárico y la Serranía del Interior Occidental (FIGURA 8B) a lo largo de tres transectos diferentes en las subcuencas de Guárico y Maturín, las cuales integran la Cuenca Oriental de Venezuela (FIGURA 8B). Empleando análisis de líneas sísmicas en dos dimensiones, geología de campo y las edades obtenidas en los tres transectos antes mencionados, discrimina seis fases de deformación para el norte de la placa suramericana:

1) Extensión NO-SE: ocurrida durante el TriásicoJurásico, esa extensión produce la apertura de un graben (Graben de Espino) el cual se extiende desde el occidente de la Serranía del Interior hasta la Cuenca Barinas Apure (FIGURA 8B).

2) Un margen pasivo en el cretácico que se extendía hacia el norte, que posteriormente disminuyó debido al enfriamiento. En ese momento, ocurre el aumento del nivel del mar, la transgresión y la invasión de los mares proto-caribeños en la América del Sur continental.

3) Contracción I: ocurrida durante el PaleocenoEoceno, las placas de América del Norte y del Sur comenzaron a converger en dirección NO$\mathrm{SE}$, en ese momento la convergencia de las placas era oblicua. La zona fronteriza era E-O; por lo tanto, la convergencia era muy oblicua, lo cual causó el desplazamiento de la partición en la zona alóctona más septentrional de la Cordillera de la Costa. El plegamiento y corrimiento de la cadena fue el resultado del acortamiento N-S relacionado con la componente normal de la convergencia. La componente paralela es expresada como una reactivación de fallas rumbo-deslizantes y zonas de cizallas. Sin embargo, las estructuras de deformación en la Serranía del Interior fueron formadas por el acortamiento N-S. La zona de la Falla de La Victoria (FIGURA 8C) al norte de la Serranía del Interior es la estructura más prominente en donde se han llevado a cabo importantes desplazamientos rumbo-deslizantes destrales. Debido a la significativa convergencia oblicua entre las placas Caribe y Suramérica, la colisión del arco de las Antillas Menores con Suramérica se produce de forma diacrónica produciendo levantamientos en diferentes épocas: en el Paleoceno y en el Eoceno, se generan las napas de Lara (FIGURA 8B); durante el Oligoceno $\mathrm{y}$ el Mioceno se produce exhumación importante en distintos sectores de la Serranía del Interior. Otros eventos importantes de exhumación ocurren a lo largo de la península de Paría durante el Mioceno a Pleistoceno. Las edades AFT se hacen más antiguas en la Serranía del Interior occidental, lo cual apoyaría el modelo de colisión diacrónica de acuerdo con Pérez de Armas (2005). Durante el Mioceno medio un importante evento regional produce la inversión de cuencas en la plataforma venezolana, resultando en el levantamiento de toda el área. El autor sugiere una posible obducción de las napas de Lara fue producido por el cambio de polaridad de la subducción de norte (durante al Paleógeno) a sur (durante el Neógeno).

4) La incepción del espesor sedimentario hacia el orógeno (foredeep) durante el Eoceno tardío a Oligoceno temprano fue contemporáneo con la 
extensión a lo largo de la subcuenca de Guárico. En este período fueron generadas nuevas fallas normales y reactivadas otras. Este foredeep se fue profundizando como respuesta a la carga de las napas de Lara, el Escudo de Guayana y el cinturón plegado Paleozoico. Las edades AFT para esa área indican el inicio del levantamiento y el enfriamiento desde el Oligoceno tardío al Mioceno medio.

5) Inclinación de la subcuenca de Guárico: se inclina hacia el este durante un período de levantamiento tectónico que ocurrió durante el PliocenoPleistoceno.

6) Evento Paleógeno: muchas edades AFT de las formaciones sedimentarias presentes son más jóvenes que las edades estratigráficas, lo que sugiere que para este período la deformación ya había comenzado en la parte central norte de Venezuela, mucho antes de la llegada de las Islas de las Antillas Menores y la colisión del arco insular. La convergencia entre América del Sur y del norte durante el Eoceno posiblemente generó la cuenca en la parte central este de Venezuela, como margen pasivo que después se deforma durante el Eoceno medio.

Sisson et al. (2005) reportaron 3 edades AFT y 3 ZFT a lo largo de la Cordillera de la Costa, 3 AFT y 8 ZFT a lo largo de la Serranía del Interior (FIGURA 8A). Las edades AFT pertenecientes a la parte más sur del cinturón plegado de la Serranía del Interior, y en sedimentos de la subcuenca de Guárico son ligeramente más antiguas que las encontradas en la Cordillera de la Costa. Esto puede sugerir diferencias regionales en la exhumación final a lo largo de estas tres regiones. Adicionalmente, en la Serranía del Interior se observaron edades ZFT más antiguas que la edad fósil, indicando una posible procedencia Cretácica para las capas sedimentarias Miocenas. Estos autores indican que la amplia gama de edades encontradas en la zona registra una variedad de eventos tectónicos que van desde el inicio del rift Jurásico hasta colisiones en el Cretáceo, subducción, y exhumación, seguidas de empuje terciario y más exhumación. La discriminación de estos eventos tectónicos incluye el reporte de varios cuerpos ígneos y rocas meta-ígneas dentro de una zona considerada como un mélange de subducción que pueden ser correlacionadas con eventos orogénicos ocurridos en los Apalaches, una procedencia Cretácica de los estratos sedimentarios de la Serranía del Interior, y enfriamiento y exhumación durante el Mioceno. La actividad tectónica final registrada en los datos termocronológicos pudieran estar asociados con la actividad de la Falla de San Sebastián (FIGURA 8C).

Locke y Garver (2005) reportaron 20 edades por trazas de fisión en apatitos pertenecientes a estratos Cretácicos a lo largo de la Serranía del Interior Oriental. Los autores discriminaron una disminución gradual de edades de norte a sur de aproximadamente entre 30 a 15 Ma. Esta tendencia representa la trayectoria o la ruta de enfriamiento principal, registrada por la mayoría de las muestras. Los autores basados en historias tiempo-Temperatura sugieren un modelo para la evolución tectónica de la Serranía del Interior oriental en dos etapas de deformación. Asumiendo 115 $\mathrm{km}$ de acortamiento total a una velocidad de $3,5 \mathrm{~mm} /$ año, la deformación dentro de la Serranía comenzó aproximadamente hace $45 \mathrm{Ma}$. La Etapa 1 (45-20 Ma) involucró la deformación interna de los bloques de falla a medida que se avanza hacia el sur. Las rocas fueron progresivamente levantadas y erosionadas de norte a sur. La cantidad de acortamiento durante la Etapa 1 fue de $\sim 88 \mathrm{~km}$. La Etapa 2 (20-12 Ma) involucró el empuje de una secuencia que duplica en espesor a la cadena sobrecorrida actual. La cantidad de acortamiento durante la Etapa 2 fue de $\sim 28 \mathrm{~km}$. La erosión ha continuado hasta el presente, lo que ha traído las rocas a la superficie. El acortamiento general dentro de la Serranía del Interior parece haber cesado a los 12 Ma con el establecimiento de la Falla de El Pilar (FIGURA 8C), pero las edades AFT parecieran indicar un enfriamiento relacionado con la etapa final de deformación. Es posible que la transpresión a lo largo de la Falla de El Pilar haya dado como resultado la tendencia de 3-13 Ma hacia el sur. La principal fase de empuje en la Serranía del Interior previa a la colisión de la placa caribeña con América del Sur está probablemente relacionada con la convergencia de las placas de América del Norte y del Sur, que ha sido relativamente estable desde los $50 \mathrm{Ma}$. La última fase de deformación coincide con la transpresión en la Falla de El Pilar y la transpresión entre la placa sudamericana y la placa del Caribe.

Cruz et al. (2007) reportaron 7 edades AFT y 6 edades ZFT bajo la modalidad de la técnica del detector externo a lo largo de la Península de Paria (FIGURA 8B). Las edades AFT oscilan entre los 29 Ma en el sur y los 5 Ma en el norte. Del mismo modo, las muestras de la zona norte y central arrojaron las edades más jóvenes ZFT que oscilaban entre los 5 y los $9 \mathrm{Ma}$, y la zona sur arrojó edades ligeramente superiores, de unos $13 \mathrm{Ma}$. A partir de las edades obtenidas por trazas de fisión, 
los autores estimaron un enfriamiento diacrónico de sur a norte y una tasa de enfriamiento en el rango de $16-56^{\circ} \mathrm{C} / \mathrm{Ma}$ ( 1-2 mm/año de exhumación). El hecho de que muchas de las edades de enfriamiento fueran posteriores a la transpresión (10 Ma) sugiere que la extrusión vertical impulsada tectónicamente por sí sola no puede explicar la exhumación observada en la zona de estudio. Así estos autores combinaron datos termocronológicos con datos estructurales, topográficos y de precipitación pluvial del orógeno transpresional de la Península de Paria, con la finalidad de interpretar e incluir el cinturón metamórfico de Paria como parte integral de una cuña transpresional de dos lados. Los datos estructurales y termocronológicos sugieren un patrón de exhumación similar al de un canal que es más alto en el borde retro. El lugar de exhumación en el cinturón metamórfico de Paria coincide con el área (borde retro) que tiene la máxima tasa de erosión interpretada y que probablemente está sustentada por una profunda raíz de la corteza que se formó a través de un flujo descendente significativo de material durante la transpresión anterior a los $10 \mathrm{Ma}$. Esto impulsa el levantamiento isostático, la erosión y la exhumación recientes (posteriores a los $10 \mathrm{Ma}$ ). Los autores hacen una analogía entre los orógenos transpresionales del al Península de Paria y los Alpes del Sur de Nueva Zelanda y proponen un modelo general para la evolución de estos dos orógenos transpresionales en el que hay una canalización global del material hacia arriba (extrusión) y hacia abajo (raíz) hacia el extremo posterior del sistema transpresional. Tanto la extrusión vertical como la erosión isostática pueden contribuir a la exhumación. Modelado numérico y termocinemático realizado en orogénos transpresionales han demostrado que la oblicuidad, y el ángulo de compresión ejercen un control primario sobre los procesos de exhumación y deformación que tienen lugar en las rocas de la corteza terrestre. De acuerdo con la evidencia geológica de los dos orógenos transpresionales naturales considerados en este estudio y los modelos numéricos publicados de transpresión, un componente de cizallamiento puro puede ser necesario para exhumar rocas en orógenos transpresionales. Esto produce tanto flujo ascendente (extrusión) como flujo descendente (raíz). El flujo descendente produce efectos duraderos. La exhumación erosiva isostática puede continuar mucho después de que la transpresión se interrumpa.

Finalmente, Flores et al. (2017) dataron 3 muestras pertenecientes a sedimentos modernos de los ríos Camatagua, Guárico y Orituco (FIGURA 8A) los cuales drenan la parte central de la Serranía del Interior. Los autores indican que las cuencas de Camatagua y Guárico poseen terrenos fuentes muy similares, discriminándose tres edades: Pérmico-Triásico $(250,6 \pm 29,2 \mathrm{Ma})$, Cretácico $(96,3 \pm 7,9 \mathrm{Ma})$ y Paleoceno-Eoceno $(57,7 \pm 3,3$ Ma). En contraste, la cuenca del río Orituco exhibe las mismas poblaciones de edades con un terreno fuente adicional, Jurásico (145,2 $\pm 12,4 \mathrm{Ma})$, lo cual es indicativo de los procesos de rifting ocurridos en el área. Las edades fueron combinadas con técnicas de geomorfología cuantitativa con la finalidad de discriminar los posibles agentes controladores de la evolución topográfica actual, los autores sugieren que al menos la parte central de la Serranía del Interior podría estar controlada principalmente por el clima y localmente por la tectónica.

\section{Venezuela occidental: Sierra de Perijá y Andes de Venezuela}

Shagam et al. (1984) reportaron 56 edades por trazas de fisión: 21 edades AFT, 19 ZFT y 2 SFT de 24 muestras de rocas recolectadas en la Isla de Toas (3), Sierra de Perijá (9), los Andes de Venezuela y el Macizo de Santander (12). Todas las edades AFT fueron interpretadas por los autores como edades de exhumación por enfriamiento. Los pulsos de exhumación discriminados por los autores fueron; Oligoceno tardío (27-22 Ma) en el piedemonte sureste de la Sierra de Perijá; Mioceno temprano a medio (19-14 $\mathrm{Ma}$ ) en el piedemonte occidental y (16-14 Ma) en la parte central del Macizo Santander; eventos de enfriamiento de Mioceno medio (13 Ma) en Toas; Mioceno tardío a Plioceno temprano (7-4 Ma) en el Macizo Central y Norte de Santander; y Plioceno medio (3 Ma) en la Sierra de Perijá. Los autores discriminaron una fase importante de exhumación ocurrida durante el Terciario.

Las edades ZFT reportadas por Shagam et al. (1984) oscilan entre los 50 y los $126 \mathrm{Ma}$. Los autores agregaron otras edades ZFT de los Andes de Venezuela reportadas por Kohn et al. (1984b) y establecieron que 8 o 9 edades de los Andes venezolanos (3), el Macizo de Santander (3), y Perijá ( 2 o 3) reflejan un levantamiento a finales del tiempo Cretácico-Paleoceno. Dos edades ZFT de la Isla de Toas y de la Sierra de Perijá son interpretadas por Shagam et al. (1984) como edades de cristalización de volcanes félsicos los cuales pudieran ocurrir entre 120 a $122 \mathrm{Ma}$. Las 23 edades restantes se interpretan como edades mixtas relacionadas con un borrado parcial ocurrido durante el Cretácico al Pérmico Temprano, aunque una concentración pronunciada de edades en el rango de 85 a $101 \mathrm{Ma}$ plantea la posibilidad de que se produzca algún evento tectonotérmico regional en ese momento. 


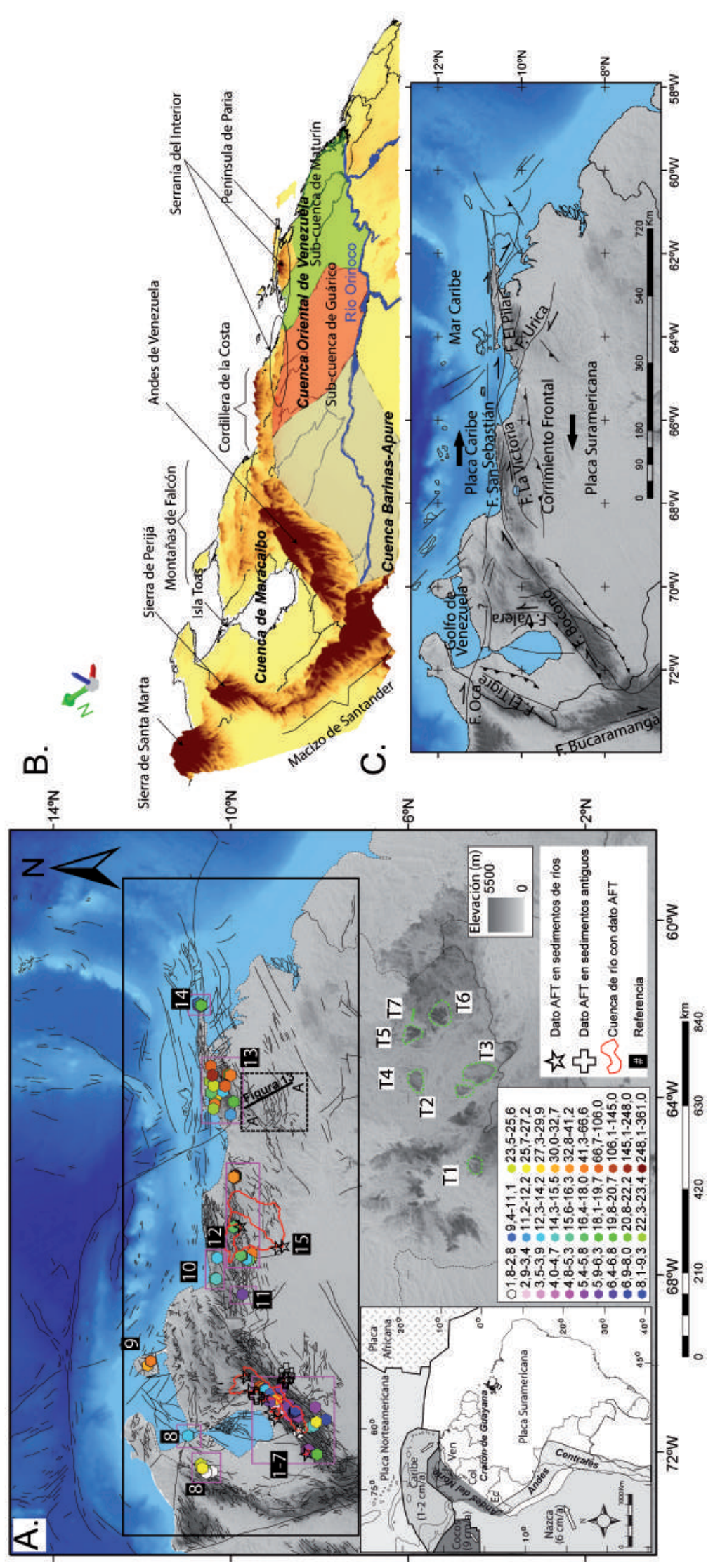

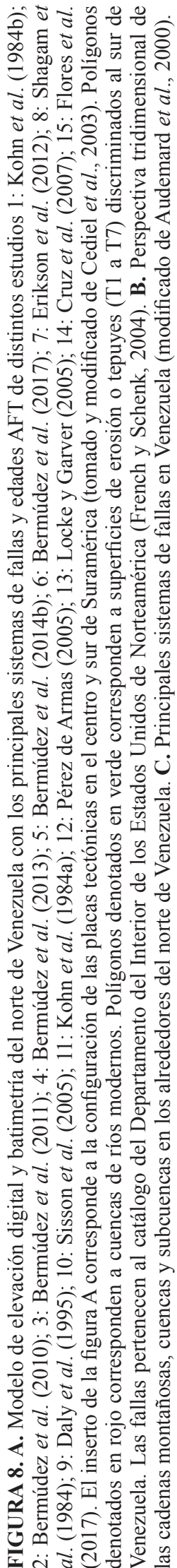


De acuerdo con Shagam et al. (1984) las regiones mencionadas anteriormente se encuentran entre los principales elementos tectónicos de un bloque continental triangular con ápice en Santa Marta, Colombia, y las fallas de Oca y Santa Marta-Bucaramanga como rumbolaterales. En esta región los autores consideran que la exhumación ocurre al final del Cretácico-Paleoceno en respuesta a la compresión noroeste-sureste que afecta a las placas caribeñas y sudamericanas.

Kohn et al. (1984b) reportaron un total de 45 edades por trazas de fisión: 22 AFT, 21 ZFT y 2 SFT de 23 muestras de rocas de los Andes de Venezuela. Nueve de las edades AFT fueron recolectadas a lo largo de un perfil vertical o transecto edad-elevación desde los 1520 a 3100 metros aproximadamente, las muestras fueron recolectadas en un mismo plutón granítico, la relación edad-elevación discriminada por los autores fue de $0,8 \mathrm{~km} / \mathrm{Ma}$. Los autores sugieren que debido a que esa tasa excede con creces las tasas aceptadas de difusión térmica en las rocas, se infiere una posible advección de isotermas.

Con base en las edades AFT, Kohn et al. (1984b) sugieren que la exhumación de los Andes venezolanos implicó en primer lugar la exhumación del margen noroeste en el Oligoceno hasta el Mioceno, seguido de una exhumación de la margen sureste a finales del Mioceno y, una exhumación de la parte central de la cadena durante el Plioceno-Pleistoceno.

Las edades ZFT oscilan de 60 a $172 \mathrm{Ma}$, con un fuerte agrupamiento en el rango entre 81 y $113 \mathrm{Ma}$. Las edades entre 60 a 72 (inicio del Paleoceno) se interpretan como edades de exhumación, lo cual según los autores estaría en conformidad con el registro estratigráfico regional. Las edades restantes fueron consideradas por Kohn et al. (1984b) como edades mixtas (sin significado geológico), las cuales pudieron haber sido afectadas por una larga residencia en la zona de desvanecimiento parcial (PAZ). Adicionalmente, dos edades SFT de 139 y 159 Ma fueron también interpretadas como edades mixtas.

Específicamente en los Andes de Venezuela, la mayoría de las muestras fechadas por Kohn et al. (1984b) estaban muy cerca de los principales sistemas de fallas. De acuerdo con Kohn et al. (1984b) esas fallas definían distintos bloques de un sistema de graben, y por lo tanto ellos interpretan las edades por trazas de fisión como puntos que representan fases intermedias (transientes) a un levantamiento o inversión de un sistema de graben, lo cual explicaría los distintos patrones reportados en las edades.

\section{Partición de la cadena andina en bloques con distintas historias tectonotermales e influencia de fallas}

Bermúdez et al. (2010) reportaron 47 edades AFT utilizando la técnica del detector externo, muchas de las edades reportadas inicialmente por Kohn et al. (1984b) fueron revisadas. La comparación de rasgos topográficos, ubicación de fallas activas y distribución de edades permitió discriminar al menos 7 bloques tectónicos (Escalante, Cerro Azul, Trujillo, Caparo, Sierra Nevada, Sierra La Culata y El Carmen) con distintas tasas de exhumación e historias tiempoTemperatura. La FIGURA 9A muestra la partición de la cadena andina en los bloques antes mencionados, todos los nombres de los bloques fueron denotados por sus siglas en inglés, con la finalidad de mantener la notación de Bermúdez et al. (2010). Así, el bloque Cerro Azul (CATB) ubicado en el flanco surandino limitado por el corrimiento SE y por el sistema de fallas de Caparo posee edades entre 2 a $10 \mathrm{Ma}$, y una longitud promedio de trazas de fisión cercana a los $13 \mu \mathrm{m}$, aunque para este bloque la mayoría de las edades se encuentran entre 5 a $7 \mathrm{Ma}$, en algunos sitios cercanos a la Falla de Caparo (CFS; FIGURA 9) se reportan edades cercanas a los $2 \mathrm{Ma}$. El bloque de Caparo (CB) posee edades entre 15 a $28 \mathrm{Ma}$, y longitud promedio de trazas de fisión cercano a los 12 $\mu \mathrm{m}$, los autores interpretan tales edades como periodos significativos de permanencia en dentro de la PAZ. El bloque del Escalante (EB; FIGURA 9) limitados por el corrimiento NE y el sistema de fallas de Boconó (BFS) tiene edades entre 6 a $10 \mathrm{Ma}$, con longitudes medias de trazas de $14 \mu \mathrm{m}$, los autores interpretan esto como un rápido enfriamiento a través de la PAZ. Entre los bloques Sierra La Culata (SLCB) y la Sierra Nevada (SNB) (FIGURA 9B) los autores sugieren que poseen similares edades de exhumación (5-8 Ma). Dentro del bloque de la Sierra La Culata (SLCB) se reportan edades entre 2 a $3 \mathrm{Ma}$, pero tal distribución de edades está restringida a un ramal de la Falla de Boconó y una traza principal de la misma. Así, Bermúdez et al. (2010) en concordancia con Kohn et al. (1984b) mantienen el nombre de esta área como Bloque El Carmen (ECB). El bloque de Trujillo reporta edades entre 10 a $145 \mathrm{Ma}$ hacia el norte del bloque, los autores sugieren que la alta dispersión de edades encontradas en este bloque indicaría la acreción de diferentes terrenos producto del desplazamiento destral de la Falla de Boconó (FIGURAS 8C y 9), las longitudes medias de trazas de fisión son cercanas a los $11 \mu \mathrm{m}$, lo cual indica un paso lento de las muestras a través de la PAZ. 
Las relaciones edad-elevación para cada uno de los bloques antes mencionados es resumida en las FIGURAS 9B y 9C, se observa en éstas que el tiempo en el cual se produce la exhumación ha sido variable y depende del bloque tectónico, de manera que los Andes de Venezuela constituye una cadena cuya exhumación comenzaría antes de $40 \mathrm{Ma}$, lo cual es compatible con los resultados de Kohn et al. (1984b).
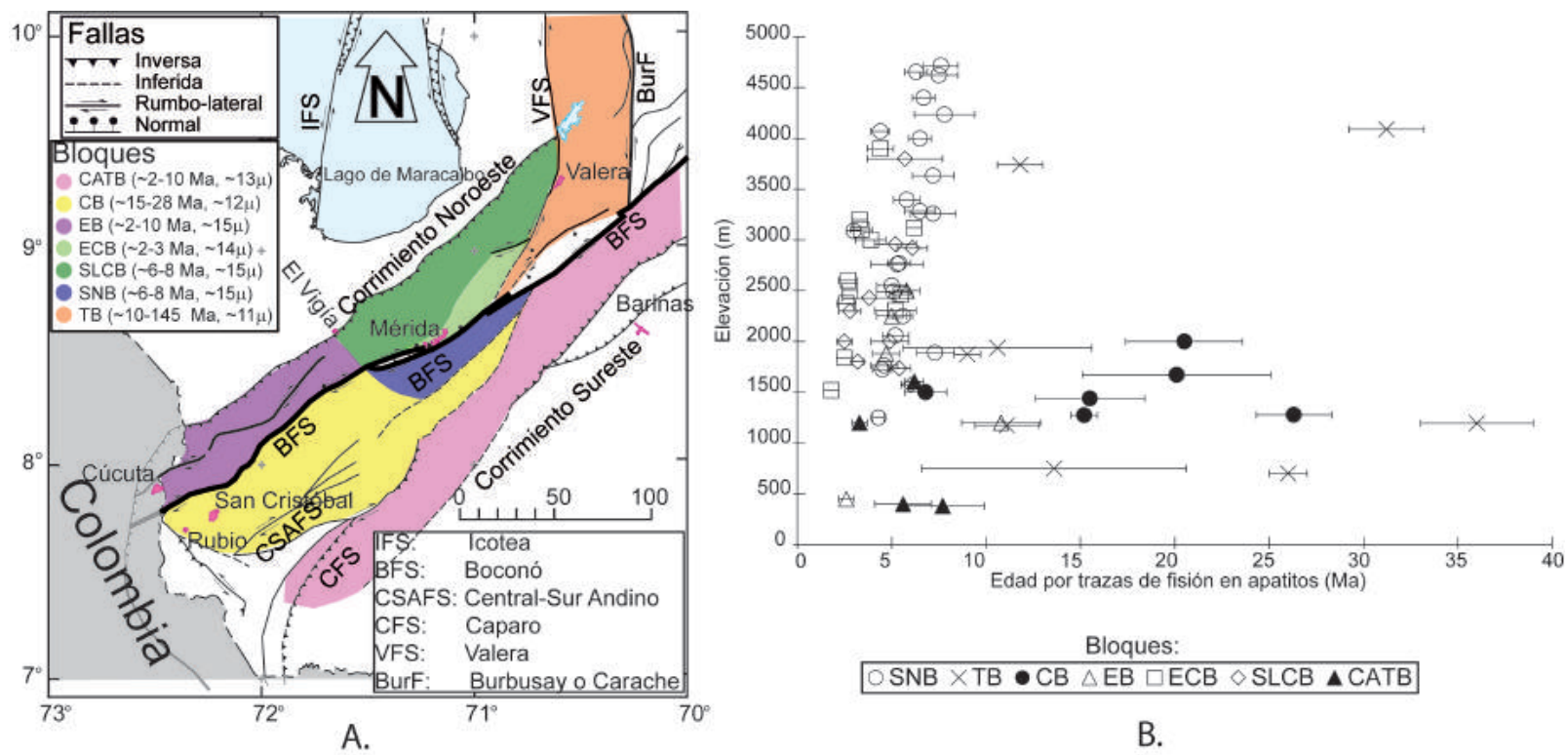

B.

FIGURA 9. A. Distintos bloques tectónicos delimitados por fallas presentes en Los Andes de Venezuela: bloque Cerro Azul (CATB), bloque de Caparo (CB), bloque El Escalante (EB), bloque El Carmen (ECB), bloque de la Sierra La Culata (SLCB), bloque de la Sierra Nevada (SNB), y bloque de Trujillo (TB). Los números entre paréntesis situados al lado de la abreviación de cada bloque corresponde con las edades y la longitud promedio de trazas de fisión encontradas en ese bloque. B. Relaciones edad-Elevación encontradas en los bloques antes mencionados (modificado de Bermúdez et al., 2010, 2014b).

La termocronología por AFT y el análisis topográfico de distintos bloques tectónicos realizado por Bermúdez et al. (2010) documentan el levantamiento y la exhumación de bloques tectónicos individuales en los Andes venezolanos. Fases de enfriamiento rápido y exhumación erosiva como consecuencia del levantamiento de la superficie tectónica y desarrollo de alto relieve durante el Mioceno tardío y el Plioceno tardío en la parte central del orógeno contrastan con las antiguas historias de exhumación de las partes noreste y suroeste de los Andes venezolanos, donde la exhumación se remonta a los tiempos del Oligoceno. El inicio de la exhumación en el Oligoceno es consistente con los registros de la Cordillera Oriental de Colombia (Parra et al., 2009) y la Sierra de Perijá (Shagam et al., 1984). La exhumación en la parte central de los Andes venezolanos (Mérida Andes) y sus flancos es el resultado de la transpresión del Mioceno tardío al Plioceno. Un importante levantamiento superficial y exhumación en el bloque SNB hace más de $8 \mathrm{Ma}$ es concordante con la reorganización fluvial registrada. por el registro sedimentario durante este período (Hoorn et al., 1995). Una segunda fase de levantamiento y exhumación afectó a los bloques ECB y EB durante el Mioceno-
Plioceno tardío. Los patrones espaciales y temporales de exhumación en los Andes venezolanos de acuerdo con Bermúdez et al. (2010) fueron fuertemente controlados por los sistemas preexistentes de fallas que delimitan los principales bloques tectónicos, como es el caso de muchos cinturones de montaña transpresionales en otros lugares. El rol de la herencia tectónica y de fallas preexistentes ha sido identificado también en cuencas de la Cordillera Oriental de Colombia (Ramírez-Arias et al., 2012).

Bermúdez et al. (2011) corroboran el comportamiento diferencial en los patrones de exhumación de la cadena para dos bloques centrales (ECB y SNB; FIGURA 9) mediante modelado numérico termocinemático 3D (PeCube, Braun, 2003; Braun et al., 2006, 2012). Para el ECB ya existía la estimación de la relación edadelevación de $\sim 0,8 \mathrm{~km} / \mathrm{Ma}$ discriminado por Kohn et al. (1984b). Para el bloque SNB, Bermúdez et al. (2011) realizan un perfil vertical en el flanco surandino, la muestra de menor elevación fue recolectada a los 1250 $\mathrm{m}$, mientras la de mayor elevación es de $4718 \mathrm{~m}$, para una diferencia de $3468 \mathrm{~m}$. Los resultados obtenidos en esa investigación permitieron comparar las 
velocidades y las edades de exhumación sobre ambos bloques, los principales resultados sugieren que el SNB ha experimentado al menos dos diferentes pulsos de exhumación, una rápida $(\sim 1,5 \mathrm{~km} / \mathrm{Ma})$ que pudo haberse iniciado desde el Mioceno temprano, y un segundo pulso que continua actualmente y que sugiere una disminución de la tasa de exhumación a $\sim 0,3 \mathrm{~km} /$ $\mathrm{Ma}$, desde hace $4 \mathrm{Ma}$. En contraste, ECB situado en la Sierra La Culata, ha aumentado su tasa de exhumación a $\sim 1,5 \mathrm{~km} /$ Ma desde los últimos 3,5 Ma (FIGURA 9).

Además de la partición de la cadena en diferentes bloques tectónicos que son exhumados independientemente a diferentes velocidades y que posterior o sincrónicamente fueron desplazados por el movimiento transcurrente dextral del sistema de fallas de Boconó, se suma el hecho de que gran parte de la deformación actual está siendo afectada por un proceso de escape a gran escala, en la cual todo el bloque de los Andes del Norte de Suramérica (FIGURA 8) está trasladándose hacia el noroeste producto del empuje que genera el itsmo de Panamá y la Placa del Caribe (Dhont et al., 2005; Backé et al., 2006; Egbue y Kellogg, 2010).

Las predicciones de los modelos termocinemáticos son válidas para otros termocronómetros aplicados como la técnica de (U-Th)/He en apatitos y circones. Bermúdez et al. (2014a) realizan un muestreo a lo largo de la traza principal de la Falla de Boconó, y empleando los métodos de datación trazas de fisión en apatitos y (U-Th)/He en circones (ZHe) reportan 9 nuevas edades AFT y 6 edades (ZHe) la mayoría de las muestras tomadas a lo largo de la traza principal de la Falla de Boconó, y una de las muestras pertenecientes al flanco surandino (Formación Pagüey). Los autores sugieren que las nuevas edades AFT demuestran que está falla ha estado activa desde hace $30 \mathrm{Ma}$, con una

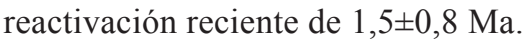

\section{Discriminación de los controles tectónicos y/o climáticos sobre los patrones de exhumación en Los Andes de Venezuela}

Bermúdez et al. (2013) reportaron 8 edades AFT pertenecientes a sedimentos recientes de cuencas hidrográficas a lo largo de los Andes de Venezuela (cuencas 1 a 7 y 13 en la FIGURA 10), en ese estudio multidisciplinario, las edades fueron convertidas a tasas de erosión a largo plazo y comparadas con algunas estimaciones de tasas de erosión actual (o stream power index), también ese trabajo muestra una compilación de datos sísmicos desde 1911 a 2011, que fueron convertidos a energía y deformación sísmica.
Se emplearon tasas de precipitación y estimaciones de relieve que fueron comparados entre sí mediante análisis de correlaciones con la finalidad de discriminar el fuerte control tectónico y el débil control climático sobre los patrones de exhumación de Los Andes de Venezuela.

Actualmente, en el Laboratorio de Termocronología de la UCV se están estudiando al menos 18 cuencas hidrográficas de diversos tributarios a lo largo de la cuenca principal del río Orinoco (FIGURA 10), el objetivo de ese proyecto es tratar de establecer las relaciones existentes entre exhumación de orógenos y procesos de desviación/reorganización de las redes de drenajes, paleogeografía. Los resultados preliminares han sido reportados en Bustos y Bermúdez (2017) y en Bermúdez (2015) quienes sugieren diversos controles a escala local y un rejuvenecimiento en la topografía de los terrenos circundantes. Bermúdez (2015) reporta 24 edades ZFT en sedimentos modernos pertenecientes a las cuencas de los ríos: Agua Viva, Chama, Chejendé de la zona central de los Andes de Venezuela, Coloncito Mimbós, San Pedro, y Tucaní del flanco norandino, Boconó, Canaguá, Caparo, Capitanejo, Parángula, Santo Domingo y Uribante del flanco surandino, Camatagua, Guárico, Orituco, Peña y Tucuragua de la Cordillera de la Costa, subcuenca de Guárico y Serranía del Interior, y Apure, Cataniapo, Meta, Orinoco y Parguaza los cuales drenan parte del flanco surandino, el flanco este de la Cordillera Oriental de Colombia y parte del Cratón de Guayana.

\section{Uso de la termocronología detrítica para refinar edades de depositación en cuencas de Venezuela}

Erikson et al. (2012) reportaron 6 edades AFT pertenecientes a sedimentos sinorógenicos del flanco surandino, a lo largo de una sección estratigráfica de $1100 \mathrm{~m}$, las edades oscilan de $11,2 \pm 1,3$ a 13,8 $2,0 \mathrm{Ma}$. El modelado de historias termales usando las edades AFT obtenidas, la distribución de la longitud de trazas de fisión y datos de reflectancia de vitrinita para Formación Río Yuca inferior indica que: 1) que la exhumación del terreno fuente ocurría entre 12 a $14 \mathrm{Ma}, 2$ ) los granos de apatito alcanzaron la superficie a los 9-10 Ma, 3) el enterramiento de los granos en la sección sedimentaria expuso los granos a temperaturas tan altas como $80^{\circ} \mathrm{C}, \mathrm{y}$ 4) el área pudo haber sufrido fue una nueva exhumación a los 2-3 Ma. De acuerdo con Erikson et al. (2012) la depositación de la Formación Río Yuca es contemporánea con una migración de la cuenca Barinas-Apure hacia el frente de deformación. 


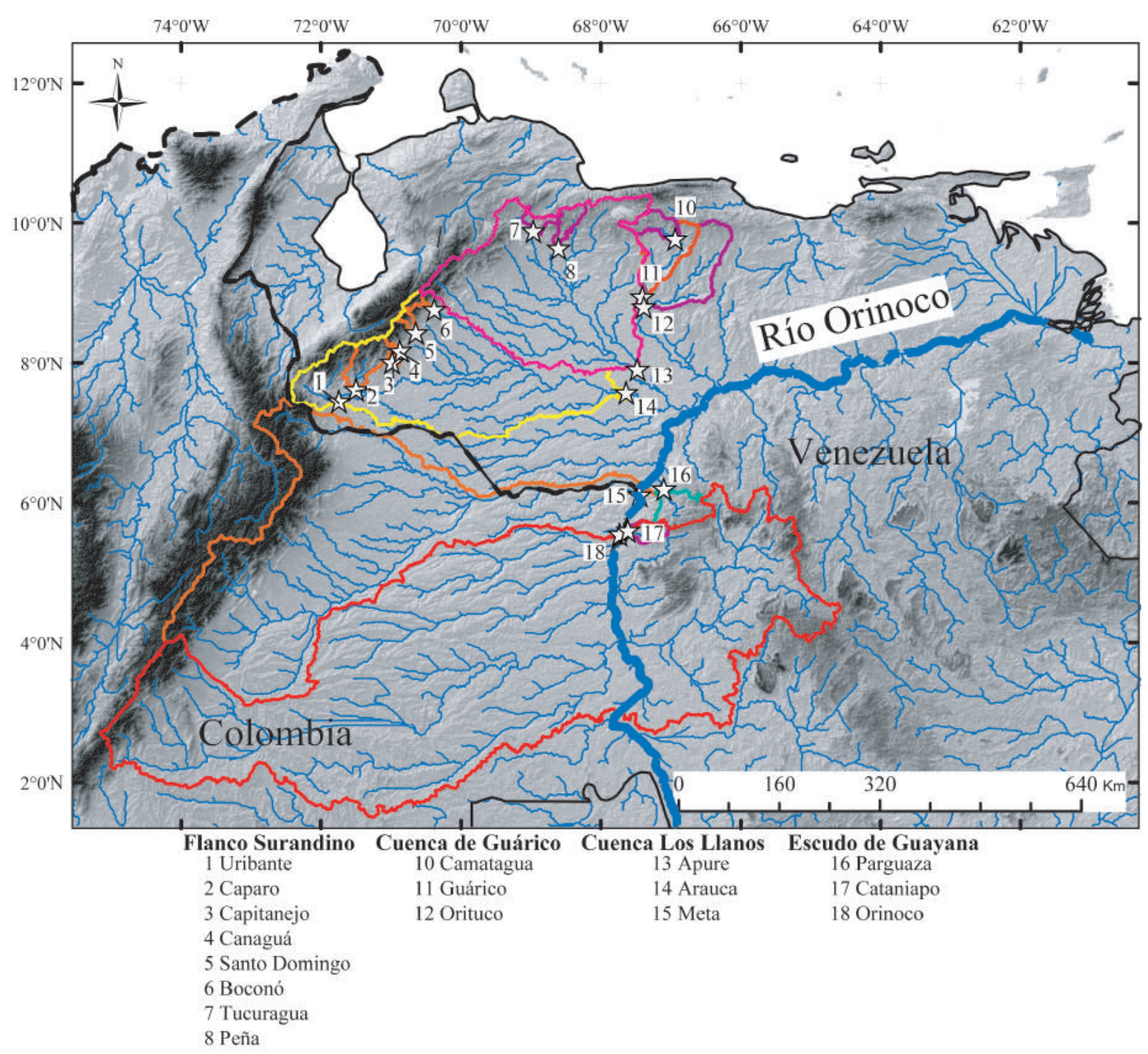

FIGURA 10. Principales cuencas hidrográficas en estudio con la finalidad de discriminar las relaciones existentes entre clima, relieve y tectónica en los orógenos circundantes a las mismas (tomado de Bermúdez, 2015).

Bermúdez et al. (2017), bajo la misma filosofía que Erikson et al. (2012) y los estudios mencionados anteriormente para otras áreas como la sub-cuenca de Guárico y la Serranía del Interior (Pérez de Armas, 2005; Locke y Garver, 2005), usan la termocronología detrítica en areniscas pertenecientes a los dos miembros de la Formación Betijoque (Miembros Vichú y San Alejos) en la cuenca de Maracaibo en el flanco norandino (FIGURA 8B) y las formaciones Pagüey, Parángula y Río Yuca en la cuenca Barinas-Apure en el flanco surandino para refinar las edades estratigráficas, pues suponen basados en algunos datos de pirólisis RockEval que las edades no han sido reseteadas por eventos tectonotermales. Así, la edad termocronológica de la población más joven representa una aproximación a la edad estratigráfica pues el área fuente se exhumaba rápidamente, como se verifica con estudios independientes de termocronología in situ o bedrock en la parte central de los Andes venezolanos (Kohn et al., 1984b; Bermúdez et al., 2010, 2011).

Una comparación en términos de edades AFT para los sedimentos sinorogénicos de los flancos norandino y surandino es mostrada en la FIGURA 11, observándose que los sedimentos del flanco norandino son relativamente más jóvenes (1 a $2 \mathrm{Ma}$ ) que los del flanco surandino, esa diferencia pudiera corresponder al comportamiento asincrónico del patrón de exhumación en la parte media de la cadena (Bermúdez et al., 2011). 
A.

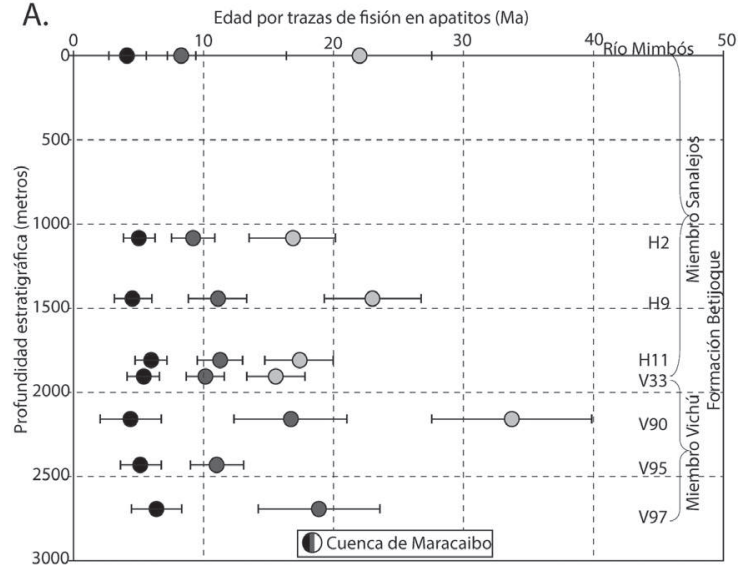

B. Edad por trazas de fisión en apatitos (Ma)

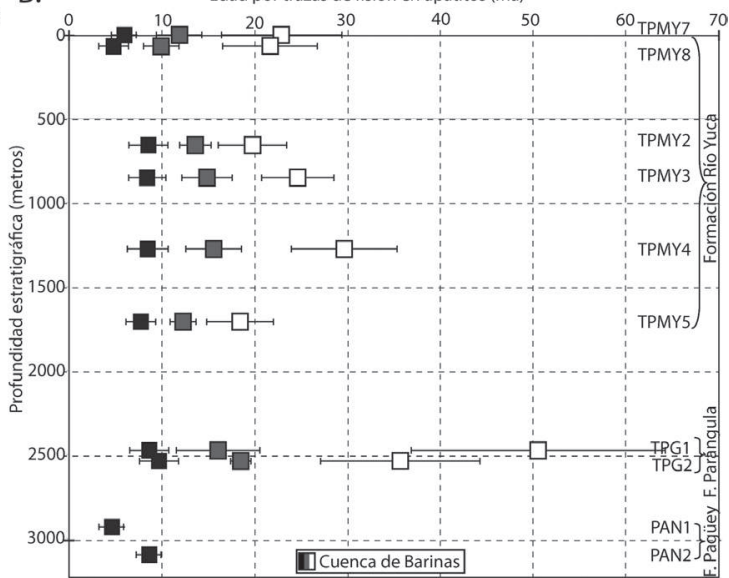

FIGURA 11. Relación entre edad AFT y profundidad de las muestras (tomado y modificado de Bermúdez et al., 2017) para: A. cuenca de Maracaibo y B. cuenca de Barinas. Los colores negro, gris y blanco representan las poblaciones 1 a 3 discriminadas, respectivamente.

Estos nuevos datos refinan la edad estratigráfica de la Formación Betijoque en el flanco norandino (antes asignada a Plioceno-Mioceno) y hoy restringida al Plioceno (FIGURA 12). La Formación Río Yuca en el flanco surandino anteriormente se extendía del Plioceno al Mioceno tardío, hoy en día Bermúdez et al. (2017) proponen desde el Plioceno al Mioceno tardío. El tope de la Formación Parángula se restringiría sólo al Mioceno tardío. Este nuevo marco cronoestratigráfico es muy importante para la geología del petróleo ya que permite establecer profundidades de las formaciones presentes en los núcleos mediante comparaciones de pozo a pozo, permite estudiar la continuidad de las formaciones geológicas de la superficie al subsuelo, así como detectar la presencia o ausencia de fallas que pudieran explicar la pérdida de reflectores o de secuencias sedimentarias.

A.

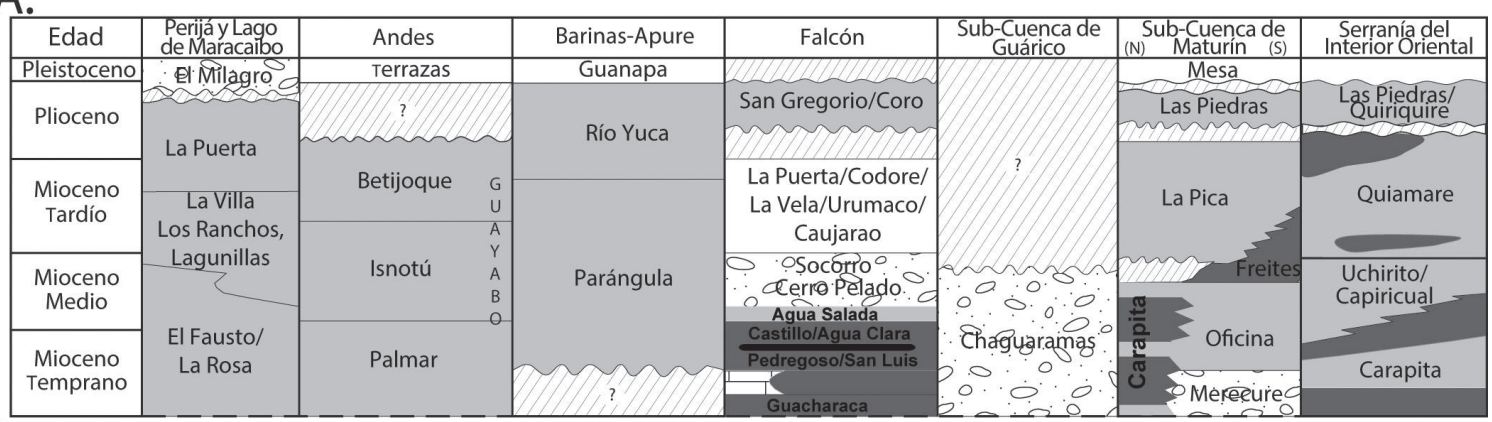

B.

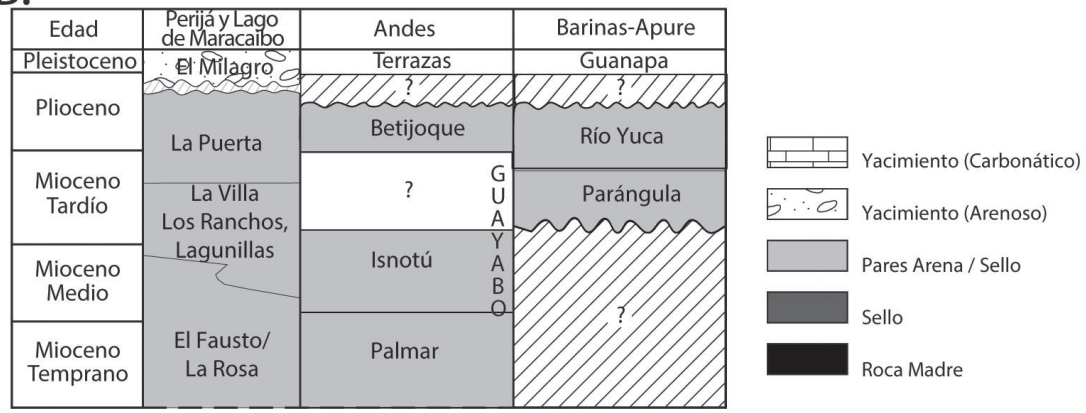

FIGURA 12. A. Cuadro de correlación entre formaciones geológicas del Neógeno (modificado de Yoris y Ostos, 1997). B. Nueva correlación entre formaciones sinorogénicas de los flancos nor y surandino propuesta después de Bermúdez-Cella (2009) y Bermúdez et al. (2017). 


\section{POSIBLES ESTUDIOS A FUTURO}

A lo largo del territorio venezolano existen muchas zonas en donde no existe una buena cobertura de datos termocronólogicos que puedan ser usados para conocer la evolución geodinámica en el tiempo de las principales cadenas montañosas, la reactivación de importantes sistemas de fallas, así como los estudios detallados de la evolución tectonotermal de las principales cuencas sedimentarias. A continuación, sugerimos algunos futuros estudios que pudieran hacerse:

Determinación de la madurez termal y correlación de formaciones geológicas a lo largo de la Faja Petrolífera del Orinoco

El antiguo esquema de correlación disponible en el Léxico Estratigráfico de Venezuela (Ministerio de Minas e Hidrocarburos, 1970) cambió al ser fechadas las formaciones sinorogénicas a ambos lados de los
Andes de Venezuela (FIGURA 12). Esos resultados resultan de gran impacto para ser aplicado a otras cuencas de Venezuela como, por ejemplo, en la cuenca de Maracaibo, en la cuenca Oriental de Venezuela, esta última comprende las subcuencas de Guárico y Maturín (FIGURA 8B), a lo largo de estas dos subcuencas se extiende la llamada Faja Petrolífera del Orinoco, un lugar donde se encuentra gran parte de las reservas de hidrocarburo pesado de Venezuela. En esa área han sido muy complicadas las dataciones por otras herramientas como bioestratigrafía o palinología, principalmente en las formaciones terciarias; por tal razón resulta importante hacer dataciones AFT y $\mathrm{U}-\mathrm{Pb}$ en zircón, siendo esta última menos susceptible al reseteo por soterramiento. La FIGURA 13 ilustra el estado actual del conocimiento que se tiene sobre la madurez termal de las principales formaciones geológicas de la Faja Petrolífera del Orinoco, pero hasta ahora esta configuración termal no ha sido calibrada usando TTF.

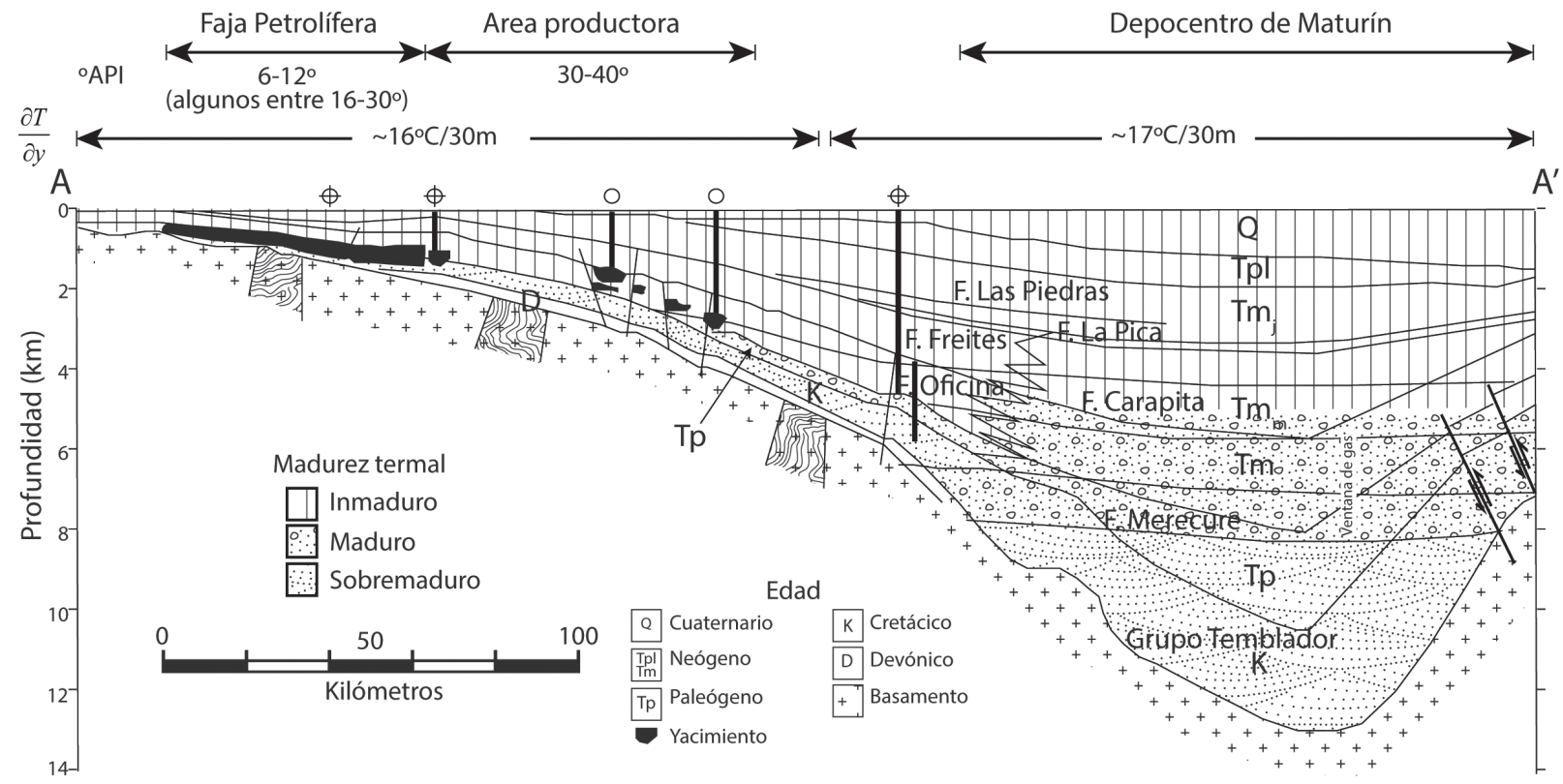

FIGURA 13. Madurez termal de las formaciones geológicas presentes en la Faja Petrolífera del Orinoco (tomada y modificada de Erlich y Barrett, 1992). El símbolo de grados $\left({ }^{\circ}\right)$ se refiere a grados API, la simbología $16^{\circ} \mathrm{C} / 30 \mathrm{~m}$ se refiere al gradiente geotérmico medido en pozos en intervalos de 30 metros.

\section{Discriminación de la historia de exhumación de la Cordillera de la Costa}

Distintos bloques tectónicos y tasas de exhumación cualitativas han sido propuestos por Urbani (2002) para la Cordillera de la Costa (FIGURA 14). Es importante reconocer las características de las distintas fallas que allí se presentan, además de realizar muestreos tipo perfiles verticales como los realizados por Bermúdez et al. (2011) con la finalidad de cuantificar realmente las tasas de exhumación. 


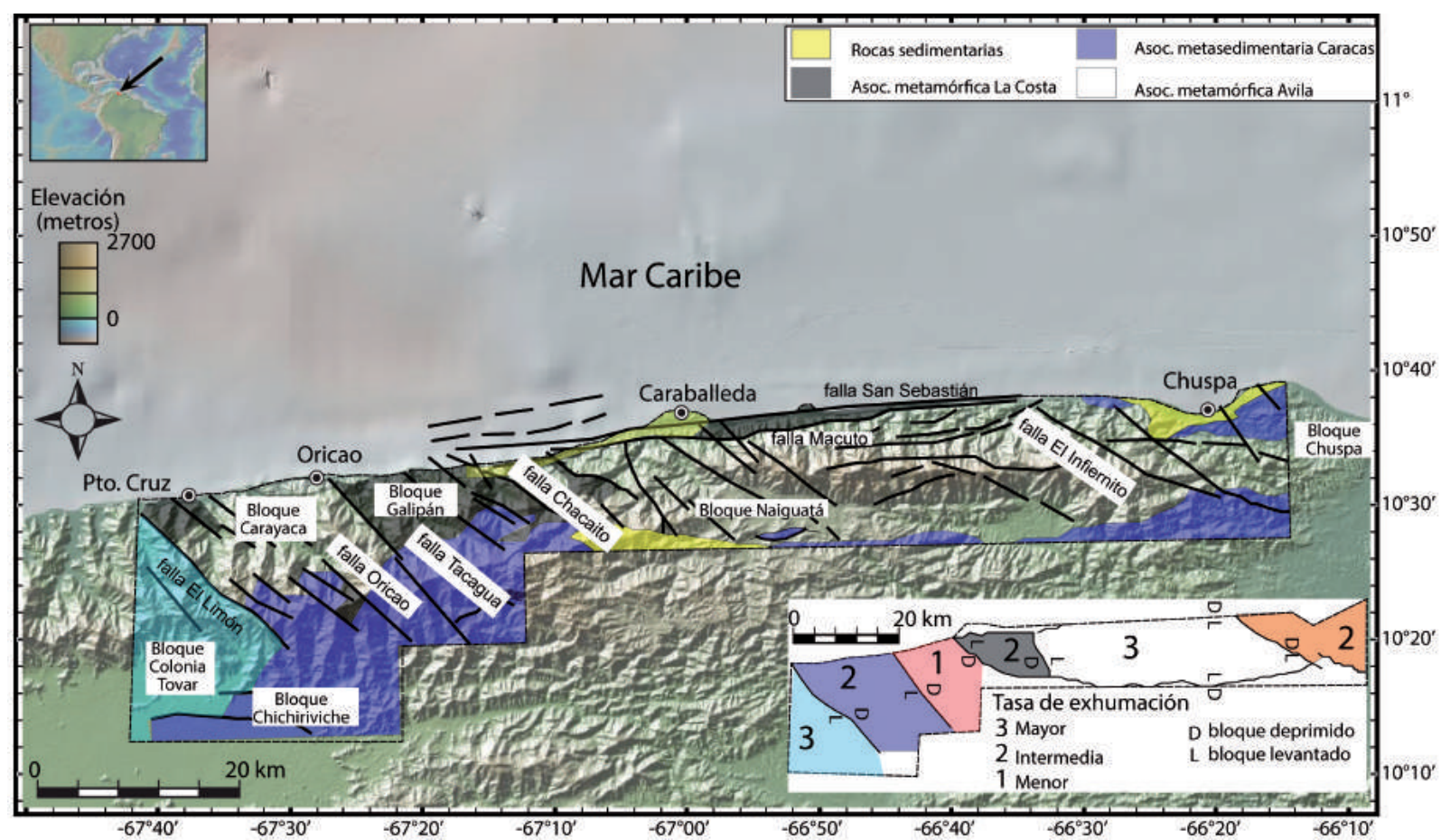

FIGURA 14. Batimetría y topografía del sector central de la Cordillera de la Costa con los distintos bloques tectónicos y tasas de exhumación cualitativas de Urbani (2002).

\section{Discriminación de la evolución topográfica del Cratón de Guayana}

Al menos 6 superficies de erosión han sido discriminadas desde el Cratón de Guayana hasta la Serranía del Interior (Schubert, 1986) basadas en observaciones de campo, mapas topográficos, análisis y modelos evaluados a nivel regional en áreas del Escudo de Guayana. Estas superficies de distintas edades van desde el rango de los 2000-2900 en su parte más alta que ocurre en el AuyanTepui hasta los 0-50 m en las planicies de inundación del río Orinoco. La TABLA 2, resume las distintas superficies de erosión discriminadas por Schubert (1986). Una posible explicación del origen de las superficies de erosión es mostrada gráficamente en la FIGURA 15.

A futuro resultaría muy importante colectar datos de termocronología AFT y (U-Th)/He en conjunto con isótopos cosmogénicos y modelado numérico para tratar de establecer la historia de formación y evolución en el tiempo de tales superficies.

TABLA 2. Superficies de Erosión del Escudo de Guayana (tomado de Schubert, 1986).

\begin{tabular}{ccc}
\hline Elevación $(\mathbf{m})$ & Nombre de la superficie de erosión & Edad \\
\hline $2000-2900$ & Auyan- tepui & Mesozoico (?) \\
$900-1200$ & Wonken & Mesozoico (?) \\
$600-700$ & Imataca & Terciario-Temprano (?) \\
$200-450$ & Caroní-Aro & Oligo-Mioceno (?) \\
$90-150$ & Llanos & Plio-Pleistoceno (?) \\
$0-50$ & Planicie de Inundación, Orinoco & Holoceno \\
\hline
\end{tabular}



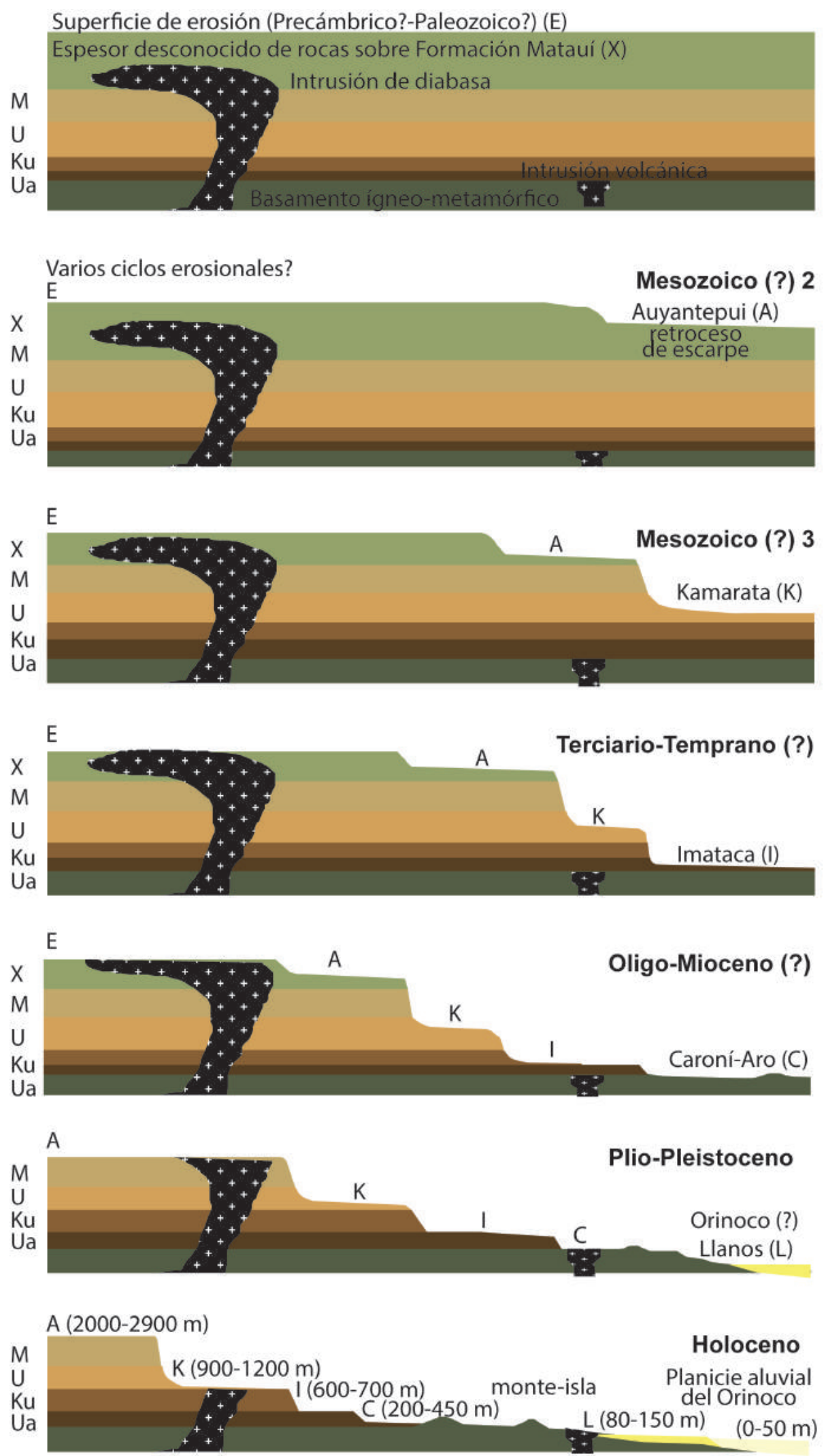

FIGURA 15. Modelo de Superficies de Erosión (tomado y modificado de Ollier y Pain, 2000). 


\section{CONCLUSIONES}

La TTF en apatitos y circones, de origen tanto in-situ en macizos cristalinos como detríticos en formaciones sedimentarias antiguas y redes fluviales modernas, tiene un amplio rango de aplicaciones que van desde las edades de reactivación de fallas, paleosismología, relación entre clima-tectónica-procesos superficiales, relaciones entre exhumación de orógenos y soterramiento en cuencas adyacentes. Esto ha permitido el estudio de la evolución geodinámica del Caribe y de Los Andes del Norte de Suramérica. Los trabajos desarrollados en Venezuela apenas constituyen un pequeño esfuerzo por entender la complicada evolución geodinámica que ha tenido el borde noroccidental de Suramérica y su relación con la Placa Caribe. Sin embargo, a futuro es necesario integrar más información y generar estudios adicionales que integren palinología, geofísica, estudios de madurez termal, termocronología, biología molecular, reflectancia de vitrinita, micropaleontología, entre otras ramas para entender las relaciones entre exhumación de orógenos, soterramiento en cuencas, formación, entrampamiento y migración de hidrocarburos desde un punto de vista del análisis de cuencas. Consideramos que es importante hacer un esfuerzo por tratar de relacionar los procesos de exhumación con la geodinámica en forma integrada a lo largo de los Andes del Norte de Suramérica.

\section{AGRADECIMIENTOS}

A los Proyectos DIN-SGI-2417 y SGI-2666 de la Universidad Pedagógica y Tecnológica de Colombia (UPTC). También los autores agradecen a Rosa Jiménez, Charles Viana, Surisaday Arcia, Michael Schmitz, Matthias Bernet, Barry Kohn, Peter van der Beek, Carina Hoorn y Suzette Flantua por el soporte proporcionado durante estos años. Agradecemos a Mauricio Parra-Amézquita y Sergio Andrés RestrepoMoreno por sus comentarios y revisión detallada los cuales ayudaron a mejorar el presente trabajo.

\section{REFERENCIAS}

Audemard, F.A., Machette, M.N., Cox, J.W., Dart, R.L., and Haller, K.M. (2000). Map and database of Quaternary faults in Venezuela and its offshore regions. U.S. Geological. Survey, Open-File Report 00-018.
Backé, G., Dhont, D., and Hervouët, Y. (2006). Spatial and temporal relationships between compression, strike-slip and extension in the Central Venezuelan Andes: Clues for Plio-Quaternary tectonic escape. Tectonophysics, 425(1-4), 25-53. doi: 10.1016/j. tecto.2006.06.005.

Bermúdez,M.A.(2015). Aplicaciones de la termocronología de baja temperatura, modelado termocinemático y geomorfología cuantitativa en el establecimiento de la evolución geodinámica de un sector del norte de Suramérica. Memoria Integrativa, 115 p. Trabajo de Ascenso a Profesor Titular, Escuela de Geología, Minas y Geofísica, Facultad de Ingeniería, Universidad Central de Venezuela.

Bermúdez, M., Alson, P., y Mora, J. (2004). Cálculo de incertidumbres en la edad proporcionada por el método de huellas de fisión y la equivalencia entre las distintas fórmulas. Revista de la Facultad de Ingeniería, 19(1), 119-123.

Bermúdez, M., Alson, P., y Mora, J. (2005). Ecuación fundamental de la edad para la datación de material rocoso y su adaptación a la ecuación práctica para el método de huellas de fisión. Revista de la Facultad de Ingeniería, 20(2), 95-103.

Bermúdez, M.A., Hoorn, C., Bernet, M., Carrillo, E., van der Beek, P.A., Garver, J.I., Mora, J.L., and Mehrkian, K. (2017). The detrital record of lateMiocene to Pliocene surface uplift and exhumation of the Venezuelan Andes in the Maracaibo and Barinas foreland basins. Basin Research, 29(S1), 370-395. doi: 10.1111/bre.12154.

Bermúdez, M.A., Kohn, B.P, van der Beek, P.A., Bernet, M., O'Sullivan, P.B., and Shagam, R. (2010). Spatial and temporal patterns of exhumation across the Venezuelan Andes: Implications for Cenozoic Caribbean geodynamics. Tectonics, 29(5). doi: 10.1029/2009TC002635.

Bermúdez, M.A., van der Beek, P., and Bernet, M. (2011). Asynchronous Miocene-Pliocene exhumation of the central Venezuelan Andes. Geology, 39(2), 139-142. doi: 10.1130/G31582.1.

Bermúdez, M.A., van der Beek, P.A., and Bernet, M. (2013). Strong tectonic and weak climatic control on exhumation rates in the Venezuelan Andes. Lithosphere, 5(1), 3-16. doi: 10.1130/L212.1. 
Bermúdez, M.A., Kohn, B., van der Beek, P., y Bernet, M. (2014a). Patrones de exhumación de los Andes venezolanos: Un aporte de la termocronología y de la modelación numérica termocinemática 3D. Acta Científica, 65(2),17-27.

Bermúdez, M.A., van der Beek, P.A., Bernet, M., Kohn, B.P., Hoorn, C., y Carrillo, E. (2014b). Revisión de estudios termocronológicos por trazas de fisión en apatitos a lo largo de Los Andes de Venezuela y su relación con el contexto geodinámico Caribe. En: M. Schmitz, F. Audemard, F. Urbani (eds.). El límite noreste de la placa sudamericana estructuras litosféricas de la superficie al manto (pp. 305-231). Universidad Central de Venezuela.

Bermúdez-Cella, M.A. (2009). Cenozoic exhumation patterns across the Venezuelan Andes: insights from fission-track thermochronology. PhD. Thesis, Université Joseph-Fourier-Grenoble I, Grenoble, France.

Bostick, N., Cashman, S., McCulloh, T., and Waddell, C. (1978). Gradients of vitrinite reflectance and present temperature in the Los Angeles and Ventura basins, California. Symposium in Geochemistry: Low Temperature Metamorphism of Kerogen and Clay Minerals, California, USA.

Braun, J. (2003). Pecube: a new finite-element code to solve the $3 \mathrm{D}$ heat transport equation including the effects of a time-varying, finite amplitude surface topography. Computers and Geosciences, 29(6), 787-794. doi: 10.1016/S0098-3004(03)00052-9.

Braun, J., van der Beek, P., and Batt, G. (2006). Quantitative thermochronology: Numerical methods for the interpretation of thermochronological data. Cambridge: Cambridge University Press. doi: 10.1017/ CBO9780511616433.

Braun, J., van der Beek, P., Valla, P., Robert, X., Herman, F., Glotzbach, C., Pedersen, V., Perry, C., Simon-Labric, T., and Prigent, C. (2012). Quantifying rates of landscape evolution and tectonic processes by thermochronology and numerical modeling of crustal heat transport using PECUBE. Tectonophysics, 524-525, 1-28. doi: $10.1016 /$ j.tecto.2011.12.035.

Bustos, X., y Bermúdez, M. (2017). Determinación y comparación de índices de erosión teóricos en cuencas del flanco surandino venezolano, apoyado en sistemas de información geográfica y programación Python. Terra. Nueva Etapa, 33(53), 105-122.

Cediel, F., Shaw, R.P., and Cáceres, C. (2003). Tectonic assembly of the Northern Andean Block. In: C. Bartolini, R.T. Buffler, J.F. Blickwede (eds.). The Circum-Gulf of Mexico and the Caribbean: Hydrocarbon habitats, basin formation, and plate tectonics. (pp. 815-848). Vol. 79, AAPG Memoir.

Chew, D.M., and Spikings, R.A. (2015). Geochronology and thermochronology using apatite: time and temperature, lower crust to surface. Elements, 11(3), 189-194. doi: 10.2113/ gselements.11.3.189.

Cruz, L., Fayon, A., Teyssier, C., and Weber, J. (2007). Exhumation and deformation processes in transpressional orogens: The Venezuelan Paria peninsula, SE Caribbean-South American plate boundary. In: S.M. Roeske, A.B. Till, D.A. Foster, J.C. Sample (eds.). Exhumation Associated with Continental Strike-Slip Fault Systems (pp. 149165). vol. 434, Geological Society of America.

Daly, M.C., Hegarty, K.A., Duddy, I.R., and Green, P.F. (1995). Structural development and tectonic history of the Venezuelan Andean Ranges and Associated basins: A reconnaissance study using thermal history data. AAPG International Conference, Nice, France.

Dhont, D., Backé, G., and Hervouët, Y. (2005). Plio-Quaternary extension in the Venezuelan Andes: Mapping from SAR JERS imagery. Tectonophysics, 399(1-4), 293-312. doi: 10.1016/j.tecto.2004.12.027.

Dodson, M.H. (1973). Closure temperature in cooling geochronological and petrological systems. Contributions to Mineralogy and Petrology, 40(3), 259-274. doi: 10.1007/BF00373790

Dow, W.G. (1977). Kerogen studies and geological interpretations. Journal of Geochemical Exploration, 7, 79-99. doi: 10.1016/03756742(77)90078-4.

Dow, W.G. (1978). Petroleum source beds on continental slopes and rises. AAPG Bulletin, 62(9), 1584-1606. 
Egbue, O., and Kellogg, J. (2010). Pleistocene to Present North Andean "escape". Tectonophysics, 489(14), 248-257. doi: 10.1016/J.TECTO.2010.04.021.

Ehlers, T.A. (2005). Crustal thermal processes and the interpretation of thermochronometer data. Reviews in Mineralogy and Geochemistry, 58(1), 315-350. doi: 10.2138/rmg.2005.58.12.

Erikson, J.P., Kelley, S.A., Osmolovsky, P., and Verosub, K.L. (2012). Linked basin sedimentation and orogenic uplift: The Neogene Barinas basin sediments derived from the Venezuelan Andes. Journal of South American Earth Sciences, 39, 138-156. doi: 10.1016/j.jsames.2012.04.002.

Erlich, R.N., and Barrett, S.F. (1992). Petroleum geology of the Eastern Venezuelan Foreland Basin. In: R.W. Macqueen, D.A. Leckie (eds.). Foreland Basins and Fold Belts (pp. 341-362). AAPG Memoir 55.

Farley, K.A. (2002). (U-Th)/He dating: techniques, calibrations, and applications. Reviews in Mineralogy and Geochemistry, 47(1), 819-844. doi: 10.2138/rmg.2002.47.18.

Faure, G. (1986). Principles of isotope geology. 2nd Edition. New York: John Wiley \& Sons.

Fleischer, R.L., Price, R.B., and Walker, R.M. (1969). Nuclear tracks in solids. Scientific American, 220(6), 30-39. doi: 10.1038/ scientificamerican0669-30.

Flores, M., Bermúdez, M.A., Bustos, X., Bernet, M., Viana, C., Arcia, S., y Schmitz, M. (2017). Análisis de los procesos controladores en la evolución topográfica de la subcuenca del río Guárico, Venezuela: mediante métodos multiherramientas. Terra. Nueva Etapa, 33(54), 71-106.

French, C.D., and Schenk, C.J. (2004). Faults of the Caribbean Region (flt6bg): Open-File Report OFR-97-470-K, U.S. Geological Survey, Central Energy Resources Team, Denver, Colorado. Consultado el 01 de octubre de 2018. https:// catalog.data.gov/dataset/faults-of-the-caribbeanregion-flt6bg.

Galbraith, R.F. (2005). Statistics for fission track analysis. Boca Raton: Chapman \& Hall/CRC Press. doi: 10.1201/9781420034929.
Gallagher, K., Brown, R., and Johnson, C. (1998). Fission-track analysis and its applications to geological problems. Annual Review of Earth and Planetary Sciences, 26(1), 519-572. doi: 10.1146/ annurev.earth.26.1.519.

Gleadow, A., Duddy, I.R., Green, P. F., and Lovering, J. (1986). Confined fission track lengths in apatite: a diagnostic tool for thermal history analysis. Contributions to Mineralogy and Petrology, 94(4), 405-415. doi: 10.1007/BF00376334.

Hood, A., Gutjahr, C.C., and Heacock, R.L. (1975). Organic metamorphism and the generation of petroleum. AAPG Bulletin, 59(6), 986996. doi: 10.1306/83D91F06-16C7-11D7$8645000102 \mathrm{C} 1865 \mathrm{D}$.

Hoorn, C., Guerrero, J., Sarmiento, G.A., and Lorente, M.A. (1995). Andean tectonics as a cause for changing drainage patterns in Miocene northern South America. Geology, 23(3), 237-240. doi: 10.1130/0091-7613(1995)023<0237:ATAACF $>2$ .3.CO;2.

Hurford, A.J. (2019). An historical perspective on Fission-Track Thermochronology. In: M.G. Malusà, P.G. Fitzgerald (eds.). Fission-Track Thermochronology and its application to geology (pp. 3-23). Cham: Springer.

Green, P.F., Duddy, I.R., Gleadow, A.J., Tingate, P., and Laslett, G. (1986). Thermal annealing of fission tracks in apatite: 1. A qualitative description. Chemical Geology: Isotope Geoscience Section, 59, 237-253. doi: 10.1016/0168-9622(86)900746.

Kohn, B.P, Shagam, R., and Subieta, T. (1984a). Results and preliminary implications of sixteen fissiontrack ages from rocks of the western Caribbean Mountains, Venezuela. In: W.E. Bonini, R.B. Hargraves, R. Shagam (eds). The CaribbeanSouth American Plate Boundary and Regional Tectonics (pp. 415-421). Vol. 162. Geological Society of America.

Kohn, B.P, Shagam, R., Banks, P., and Burkley, L. (1984b). Mesozoic-Pleistocene fission-track ages on rocks of the Venezuelan Andes and their tectonic implications. In: W.E. Bonini, R.B. Hargraves, R. Shagam (eds). The CaribbeanSouth American Plate Boundary and Regional 
Tectonics (pp. 365-384). Vol. 162. Geological Society of America.

Ministerio de Minas e Hidrocarburos. (1970). Léxico Estratigráfico de Venezuela, Boletín de Geología, Publicaciones Especiales, 4, 464p.

Locke, B.D., and Garver, J.I. (2005). Thermal evolution of the eastern Serranía del Interior foreland fold and thrust belt, northeastern Venezuela, based on apatite fission-track analyses. In: H.G. Avé Lallemant, V.B. Sisson (eds.). Caribbean-South American plate interactions, Venezuela (pp. 315328). Geological Society of America Special Papers, 394. doi: 10.1130/0-8137-2394-9.315.

Malusà, M.G., and Fitzgerald, P.G. (2019). FissionTrack Thermochronology and its application to geology. Cham: Springer.

Marshak, S. (2015). EARTH Portrait of a Planet. Nueva York: 5th edition, W.W Norton \& Company.

Moore, M.E., Gleadow, A.J.W., and Lovering, J.F. (1986). Thermal evolution of rifted continental margins: new evidence from fission tracks in basement apatites from southeastern Australia. Earth and Planetary Science Letters, 78(1-2), 255-270. doi: 10.1016/0012-821X(86)90066-X.

Mora, A., Horton, B.K., Mesa, A., Rubiano, J., Ketcham, R.A., Parra, M., Blanco, V., Garcia, D., and Stockli, D.F. (2010). Migration of Cenozoic deformation in the Eastern Cordillera of Colombia interpreted from fission-track results and structural relationships: Implications for petroleum systems. American Association of Petroleum Geologists Bulletin, 94(10), 15431580. doi: 10.1306/01051009111.

Mora, A., Blanco, V., Naranjo, J., Sanchez, N., Ketcham, R.A., Rubiano, J., Stockli, D., Quintero, I., Nemčok, M., Horton, B., and Dávila, H. (2013). On the lag time between internal strain and basement involved thrust induced exhumation: The case of the Colombian Eastern Cordillera. Journal of Structural Geology, 52, 96-118. doi: 10.1016/J.JSG.2013.04.001.

Ollier, C., and Pain, C., (2000). The origin of mountains. London: ROUTLEDGE, Taylor and Francis Group.
Parra, M., Mora, A., Sobel, E.R., Strecker, M.R. and González, R. (2009). Episodic orogenic front migration in the northern Andes: Constrains from low-temperature thermochronology in the Eastern Cordillera, Colombia. Tectonics, 28(4), 1-27. doi: 10.1029/2008TC002423.

Pérez de Armas, J. (2005). Tectonic and thermal history of the western Serranía del Interior foreland fold and thrust belt and Guárico Basin, north-central Venezuela: Implications of new apatite fissiontrack analysis and seismic interpretation. In: H.G. Avé Lallemant, V.B. Sisson (eds.). CaribbeanSouth American plate interactions, Venezuela (pp. 315-328). Geological Society of America Special Paper 394. doi: 10.1130/0-8137-2394-9.271.

Ramírez-Arias, J.C., Mora, A., Rubiano, J., Duddy, I., Parra, M., Moreno, N., Stockli, D., and Casallas, W. (2012). The asymmetric evolution of the Colombian Eastern Cordillera. Tectonic inheritance or climatic forcing? New evidence from thermochronology and sedimentology. Journal of South American Earth Sciences, 39, 112-137. doi: 10.1016/J.JSAMES.2012.04.008.

Schubert, C. (1986). Terrazas aluviales en el Escudo de Guayana: Informe preliminar. Acta Cientifica Venezolana, 37, 226-228.

Shagam, R., Kohn, B., Banks, P., Dasch, L., Vargas, R., Rodríguez, G., and Pimentel, N. (1984). Tectonic implications of Cretaceous-Pliocene fission track ages from rocks of the circum-Maracaibo Basin region of western Venezuela and eastern Colombia. In: W.E. Bonini, R.B. Hargraves, R. Shagam (eds.). The Caribbean-South American Plate Boundary and Regional Tectonics (pp.385412). v. 162, Geological Society of America, Memoir.

Sisson, V.B., Avé Lallemant, H.G., Ostos, M., Blythe, A.E., Snee, L.W., Copeland, P., Wright, J.E., Donelick, R.A., and Guth, L.R. (2005). Overview of radiometric ages in three allochthonous belts of northern Venezuela: Old zones, new ones and their impact on regional geology. In: H.G. Avé Lallemant, V.B. Sisson (eds.). Caribbean-South American plate interactions, Venezuela (pp. 91117). Vol. 394, Geological Society of America Special Paper. doi: 10.1130/0-8137-2394-9.91. 
Teichmüller, M., and Teichmüller, R. (1979). Diagenesis of coal (coalification). In: G. Larsen, G. Chilingar (eds). Developments in Sedimentology (pp. 207-246). Vol. 25, part A, chapter 5. Elsevier Scientific Publishing Company. doi: 10.1016/ S0070-4571(08)71074-4.

Urbani, F. (2002). La sección geológica del río Miguelena, Camurí Grande, estado Vargas: Una ventana a la geología de la Cordillera de la Costa. Guía de excursión. No. 02-1. Caracas: Ediciones Sociedad Venezolana de Geólogos.

Wagner, G., and van den Haute, P. (1992). Fission track dating. Netherlands: Kluwer Academic Publishers.
Yoris, F., and Ostos, M. (1997). Well Evaluation Conference. Caracas: Schlumberger Oilfield Services.

\begin{tabular}{c}
\hline \hline Mauricio A Bermúdez \\
ORCID: 0000-0003-0584-4790 \\
Marlene Flores \\
ORCID: $0000-0002-0246-7515$ \\
\hline \hline
\end{tabular}

Trabajo recibido: octubre 25 de 2018

Trabajo aceptado: agosto 30 de 2019 